\title{
Segmentation of Intracranial Vessels and Aneurysms in Phase Contrast Magnetic Resonance Angiography Using Multirange Filters and Local Variances
}

\author{
Max W. K. Law and Albert C. S. Chung
}

\begin{abstract}
Segmentation of intensity varying and low-contrast structures is an extremely challenging and rewarding task. In computer-aided diagnosis of intracranial aneurysms, segmenting the high-intensity major vessels along with the attached lowcontrast aneurysms is essential to the recognition of this lethal vascular disease. It is particularly helpful in performing early and noninvasive diagnosis of intracranial aneurysms using phase contrast magnetic resonance angiographic (PC-MRA) images. The major challenges of developing a PC-MRA-based segmentation method are the significantly varying voxel intensity inside vessels with different flow velocities and the signal loss in the aneurysmal regions where turbulent flows occur. This paper proposes a novel intensity-based algorithm to segment intracranial vessels and the attached aneurysms. The proposed method can handle intensity varying vasculatures and also the low-contrast aneurysmal regions affected by turbulent flows. It is grounded on the use of multirange filters and local variances to extract intensity-based image features for identifying contrast varying vasculatures. The extremely low-intensity region affected by turbulent flows is detected according to the topology of the structure detected by multirange filters and local variances. The proposed method is evaluated using a phantom image volume with an aneurysm and four clinical cases. It achieves 0.80 dice score in the phantom case. In addition, different components of the proposed method-the multirange filters, local variances, and topology-based detection-are evaluated in the comparison between the proposed method and its lower complexity variants. Owing to the analogy between these variants and existing vascular segmentation methods, this comparison also exemplifies the advantage of the proposed method over the existing approaches. It analyzes the weaknesses of these existing approaches and justifies the use of every component involved in the proposed method. It is shown that the proposed method is capable of segmenting blood vessels and the attached aneurysms on PC-MRA images.
\end{abstract}

Index Terms-Active contour models, aneurysms, intracranial, level sets, magnetic resonance angiography (MRA), segmentation, vessels.

\section{INTRODUCTION}

B RAIN aneurysms are vascular diseases caused by abnormal dilation of the cerebral arteries. The presence of brain

Manuscript received November 2, 2011; revised July 19, 2012; accepted July 26, 2012. Date of publication August 31, 2012; date of current version January 24, 2013. This work was supported in part by the K. S. Lo Foundation and the Research Grants Council of Hong Kong General Research Fund under Grant 612308 and Grant 612011. The associate editor coordinating the review of this manuscript and approving it for publication was Prof. Jose Bioucas-Dias.

The authors are with the Lo Kwee-Seong Medical Image Analysis Laboratory, Department of Computer Science and Engineering, The Hong Kong University of Science and Technology, Kowloon, Hong Kong (e-mail: max.w.k.law@gmail.com; achung@cse.ust.hk).

Digital Object Identifier 10.1109/TIP.2012.2216274 aneurysms is life-threatening as a burst aneurysm can lead to severe internal bleeding. In the past decade, there has been growing interest in the detection of brain blood vessels and aneurysms by performing angiographic analysis. McLaughlin and Noble proposed an algorithm [1] to automatically detect the aneurysmal region based on delineated vessel boundaries. Most of the studies concerning aneurysm detection focuses on computed tomographic angiography (CTA) and 3D rotational angiography (3DRA). Hernandez et al proposed a supervised geodesic active region method, which employs multiscale Gaussian derivative [2] image features and Parzen Windows to segment aneurysms [3]. Bogunović et al [4] refined a supervised geodesic active region method to efficiently and robustly segment cerebral vasculature with aneurysms. Firouzian et al incorporated different diffusion based image smoothing in the geodesic active contour model [5] for semi-automatic aneurysm segmentation [6]. Furthermore, interactive aneurysm segmentation was achieved by modeling the shape of the normal vessel on which an aneurysm is attached [7], [8].

Whereas CTA and 3DRA offer high vessel contrast and spatial resolutions, non-invasive acquisition techniques which involve neither injection nor radiation are preferred for preventive medical examination and screening of asymptomatic aneurysms. These techniques include phase contrast magnetic resonance angiographic (PC-MRA) images and time-of-flight MRA (TOF-MRA) images. The flowsensitive PC-MRA imaging technique captures vectorial flow velocity information. Owing to the blood movement, vascular structures are distinguished from others tissues in PC-MRA images. PC-MRA images yield better vessel lumen contrast relative to the neighboring structures than TOF-MRA images, in which the signal range of vessels is largely overlapped with other tissues. Readers can also refer to [4] and [9] for TOFMRA based aneurysm segmentation methods. Nonetheless, PC-MRA signal can significantly fluctuate due to blood flow velocity changes. Blood typically flows faster along arteries than inside the attached aneurysms. The aneurysmal regions therefore exhibit a considerably lower intensity than that in the arteries on which the aneurysms are attached.

In addition, vortex-like blood flows (turbulent flows) inside aneurysm domes nullify PC-MRA signals. Segmentation of aneurysms in PC-MRA is therefore extremely challenging. Chung et al demonstrated segmenting low contrast vessels and aneurysms by taking the flow orientation into account, in addition to flow velocity magnitude [10]. Due to the large shape variability, shape modeling is scarcely employed for 
saccular aneurysms [11]. This is in contrast to the detection of fusiform aneurysms, such as abdominal aortic aneurysms (AAA) which exhibit relatively simple geometry. For instance, detection of AAA was modeled as an abrupt change of radius of a vessel segment [12]. In [13], Bruijne et al described the segmentation of AAA using intensive user interaction along with shape modeling when training data is limited. Lin employed shape information to sustain the disturbance induced by AAA while performing vascular analysis [14].

In this paper, a novel method is proposed for the segmentation of intracranial aneurysms in PC-MRA images. The proposed method requires neither training nor shape assumption. The former possibly leads to training data-dependent segmentation results while the latter is possibly inflexible to handle shape variation of aneurysms. To cope with the aforementioned limitations of PC-MRA images, the proposed method makes use of a new detection filter and local variances. The new detection filter and local variances, and their previously developed variant [15] are elaborated in Section II. The detection filter is a boundary descriptor which recognizes the intensity changes of the vascular structure boundaries. It complements the local variances which are regional descriptors to reduce the effect of noise and suppress the responses induced from high intensity vessels to avoid missing low intensity aneurysms. In Section III, a multi-range scheme is presented. It handles the size variations of vasculatures in the level set based segmentation. The unsegmented aneurysm dome under the effect of severe turbulent flows is detected and reclaimed according to the topology of the segmented regions. The proposed method is evaluated and compared using an image volume acquired from a vascular phantom with an aneurysm, and four clinical PC-MRA images. Grounded on the proposed segmentation framework, the segmentation performance using various combinations of intensity discontinuity descriptors, local variances and topology-based detection are compared and studied. This also leads to the comparison between the proposed method against three existing vascular segmentation approaches. It is shown experimentally that the proposed method well suits the segmentation of vessels along with the attached low contrast aneurysms in PC-MRA images.

\section{Intensity BASED IMAGE FEATURES}

In this section, the Discontinuity-Homogeneity (DH) ratio is proposed for vascular structure detection. A discontinuityhomogeneity ratio embodies two components, an intensity discontinuity descriptor and a local intensity variance which quantifies local intensity homogeneity. Section II-A introduces the basic form of the DH ratio - Offset DH Ratio. Section II-B describes Offset and Distilled DH ratio which is more flexible to handle non-spherical structures than the offset DH Ratio does. These two DH ratios are consecutive steps leading to the development of the proposed $\mathrm{DH}$ ratio - final $\mathrm{DH}$ ratio (Section II-C).

The DH ratios are computed based on spherical regions, denoted as $S(\vec{x}, r)$ which is centered at $\vec{x}$ and has a radius $r$. The radius $r$ specifies the detection range of the DH ratios, which return strong detection responses only when $r$ coincides with the distance between $\vec{x}$ and the closest object boundary. When $r$ deviates from this distance, the DH ratio responses are suppressed. The optimal range parameter at each local position is selected by acquiring the largest $\mathrm{DH}$ ratio obtained using multiple range parameter values. This multi-range detection scheme is discussed in Section III-C.

\section{A. Offset DH Ratio}

In the formulation of the DH ratios, the intensity discontinuity descriptor provides strong responses when $r$ coincides with the distance between $\vec{x}$ and an object boundary,

$$
f_{S(\vec{x}, r)}(I)=\frac{1}{4 \pi r^{2}} \int_{\partial S(\vec{x}, r)} \frac{\partial}{\partial \hat{n}_{A}} I\left(\vec{x}-r \hat{n}_{A}\right) d A,
$$

where $\hat{n}_{A}$ is the inward normal sweeping across the sphere surface $\partial S(\vec{x}, r)$ with a radius of $r$ and $d A$ is the infinitesimal area of $\partial S(\vec{x}, r)$. The intensity variation across the boundary of $S(\vec{x}, r)$ induces detection responses. $f_{S(\vec{x} ; r)}(I)$ is positive and negative inside bright and dark objects respectively. This descriptor can be regarded as the computation of inward flux of the image gradient [16], [17]. It is widely employed on various vascular detection frameworks [16]- [21].

In the proposed method, this descriptor complements the local intensity variance computed inside $S(\vec{x} ; r)(I)$, denoted as $\operatorname{Var}_{S(\vec{x} ; r)}(I)$. On one hand, $f_{S(\vec{x}, r)}(I)$ returns strong responses only when the spherical surface touches an object boundary. On the other hand, $\operatorname{Var}_{S(\vec{x} ; r)}(I)$ is minor if the spherical surface does not overshoot the object boundary. A large ratio,

$$
\frac{f_{S(\vec{x}, r)}(I)}{\sqrt{\operatorname{Var}_{S(\vec{x}, r)}(I)}},
$$

implies $S(\vec{x} ; r)$ barely touches an object boundary without overshooting it and supplies strong detection responses for vascular detection.

Evaluating the intensity discontinuity and variance in a spherical region avoids the use of detection scale-dependent smoothing which is commonly employed in Laplacian or Hessian based segmentation schemes [2], [22]. This is beneficial as excessive smoothing hinders the detection of low contrast boundaries. Thus, Equations 1 and 2 remain sensitive to weak edges of low intensity aneurysms at a large detection scale.

An offset value is added in the denominator of the above $\mathrm{DH}$ ratio, yielding the offset $\mathrm{DH}$ ratio,

$$
\frac{f_{S(\vec{x}, r)}(I)}{\sqrt{\operatorname{Var}_{S(\vec{x}, r)}(I)}+B(I, r)},
$$

where $B(I, r)$ is the offset value which depend on the input image and the detection range. The offset value prevents a $\mathrm{DH}$ ratio from being exaggerated by an undersized local intensity variance. This offset value is negligible when $\operatorname{Var}_{S(\vec{x}, r)}(I)$ is sufficiently large. The offset $\mathrm{DH}$ ratio is analogous to our previous work [15] which assigns a constant value to $B(I, r)$ throughout the detection.

Introducing the offset term, so as to define the exaggerating condition of the DH ratio is essential. For low contrast vascular structure detection, the local intensity variance tends to be smaller (i.e. the DH ratio surges) when the detection range 


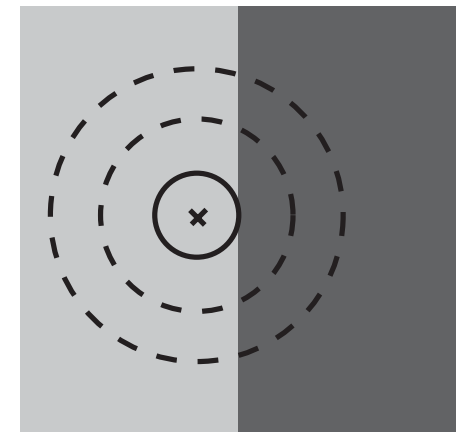

Fig. 1. Image patch with fluctuating image intensity. Local region with small detection range (solid line) is easier to avoid intensity discrepancy inside the region than those with larger detection ranges (dotted lines).

shrinks. Consequently, the detection favors small local regions. It lacks the robustness to deal with outlying voxels which generates a superior response magnitude from the differential operator $f_{S(\vec{x}, r)}(I)$. A DH ratio favors small detection ranges due to two biases, namely extrinsic bias and intrinsic bias.

1) Extrinsic Bias: The extrinsic bias occurs at the positions where the speed-dependent vessel intensity slightly fluctuates. The $\mathrm{DH}$ ratio favors a small detection range to minimize the intensity discrepancy within the local detection region (see Fig. 1). Simultaneously, the slight intensity fluctuation generates intensity discontinuity responses. The DH ratio is exaggerated and becomes over-sensitive to local intensity fluctuation, even if that fluctuation is minor as compared to the intensity changes across vessel boundaries. This possibly results in mis-recognizing the aneurysm necks as vessel boundaries.

We suggest to measure the intensity change across the major vessels and their vicinity, and determine the offset values of the $\mathrm{DH}$ ratio denominator accordingly. This encourages the $\mathrm{DH}$ ratio to sense only the major intensity changes and suppresses the adverse effect introduced by minor local intensity fluctuation. It is achieved by performing preliminary segmentation of the major vessels using solely the intensity discontinuity descriptor prior to the $\mathrm{DH}$ ratio-based segmentation. This procedure is elaborated in Section III-A. In the simplest case where extrinsic bias occurs, such as at an aneurysm neck, a local spherical region is split into two halves (hemispheres) due to intensity fluctuation. Suppose the estimated intensity change across the major vessels and their vicinity is $\rho$, the local intensity variance is $\frac{1}{2} \rho^{2}$. Other intensity changes inside a local sphere causing a local intensity variance below the value of $\frac{1}{2} \rho^{2}$ are treated as insignificant in the computation of the offset $\mathrm{DH}$ ratio. The offset value $B(I, r)$ is defined with the consideration of $\frac{1}{2} \rho^{2}$ to prohibit any insignificant intensity changes exaggerating the $\mathrm{DH}$ ratio.

2) Intrinsic Bias: The intrinsic bias is elaborated in a statistical point of view. As described in Appendix A, the expected value of the local intensity variance is strictly related to the size of the local region. A small detection range encourages a small variance value and thus, results in a large $\mathrm{DH}$ ratio. The intrinsic bias is handled by exploiting the expected value of the local intensity variance. Without considering any image prior information during low level feature extraction, suppose that the voxel intensity is independent and identically distributed (i.i.d.) variables, the expected local intensity variance computed from $n$ voxel samples is $\frac{1}{n} \operatorname{Var}_{\Omega}(I)$, where $\Omega$ is the entire image domain. For a local region having a volume size of $\frac{4}{3} \pi r^{3}$, the expected local intensity variance is $\frac{3}{4 \pi r^{3}} \operatorname{Var}_{\Omega}(I)$. If the local intensity variance is below its expected value $\frac{3}{4 \pi r^{3}} \operatorname{Var}_{\Omega}(I)$, the $\mathrm{DH}$ ratio is regarded as exaggerated. Hence, a small local region requires a larger variance to start penalizing the $\mathrm{DH}$ ratio than a large local region does owing to the detection range dependent expected local intensity variance.

3) Bias Removal: The extrinsic bias and the intrinsic bias can occur simultaneously. In such a case, we assume that the i.i.d. voxel intensity is an additive signal to the hemispherical intensity pattern. The corresponding expected local intensity variance is $\frac{3}{4 \pi r^{3}} \operatorname{Var}_{\Omega}(I)+\frac{1}{2} \rho^{2}$ for this combined intensity pattern. To eliminate both biases, the $\mathrm{DH}$ ratio at the position $\vec{x}$ is considered as exaggerated if the local intensity variance is smaller than the expected one, i.e. $\operatorname{Var}_{S(\vec{x} ; r)}(I)<$ $\frac{3}{4 \pi r^{3}} \operatorname{Var}_{\Omega}(I)+\frac{1}{2} \rho^{2}$. The offset $\mathrm{DH}$ ratio, where the local intensity variance is less influential when $\frac{3}{4 \pi r^{3}} \operatorname{Var}_{\Omega}(I)+$ $\frac{1}{2} \rho^{2}>\operatorname{Var}_{S(\vec{x} ; r)}(I)$, is formulated by specifying $B(I, r)=$ $\sqrt{\frac{3}{4 \pi r^{3}} \operatorname{Var}_{\Omega}(I)+\frac{1}{2} \rho^{2}}$, i.e.

$$
\frac{f_{S(\vec{x} ; r)}(I)}{\sqrt{\operatorname{Var}_{S(\vec{x}, r)}(I)}+\sqrt{\frac{3}{4 \pi r^{3}} \operatorname{Var}_{\Omega}(I)+\frac{1}{2} \rho^{2}}} .
$$

\section{B. Offset and Distilled DH Ratio}

In practice, the overlap between a detection spherical surface and an object boundary can be minor. This weakens the resultant magnitude of $f_{S(\vec{x} ; r)}(I)$. Although the sign of $f_{S(\vec{x} ; r)}(I)$ reliably indicates if the local region is brighter than its vicinity, the weakened responses are less distinctive for detection of vasculatures. This section elaborates the distilled intensity discontinuity descriptor, which is less sensitive to the small overlap between object boundary and the detection sphere surface. The DH ratio based on the distilled intensity discontinuity descriptor is referred to as offset and distilled $\mathrm{DH}$ ratio. The development of the distilled intensity discontinuity descriptor is grounded on expanding the term of intensity change along the sphere normal direction in Equation 1 as $\vec{\nabla} I\left(\vec{x}-r \hat{n}_{A}\right) \cdot \hat{n}_{A}$. Denote an interested detection direction by $\hat{q}_{\vec{x} ; r}^{1}$ and two orthogonal unit vectors which are also orthogonal to $\hat{q}_{\vec{x} ; r}^{1}$ by $\hat{q}_{\vec{x} ; r}^{2}$ and $\hat{q}_{\vec{x} ; r}^{3}$,

$$
\vec{\nabla} I\left(\vec{x}-r \hat{n}_{A}\right) \cdot \hat{n}_{A}=\sum_{i=\{1,2,3\}}\left(\hat{q}_{\vec{x} ; r}^{i} \cdot \vec{\nabla} I\left(\vec{x}-r \hat{n}_{A}\right)\right)\left(\hat{q}_{\vec{x} ; r}^{i} \cdot \hat{n}_{A}\right) .
$$

The intensity discontinuity descriptor stated in Equation 1 is decomposed into three orientation sensitive descriptors,

$f_{S(\vec{x} ; r)}(I)=f_{S(\vec{x} ; r)}\left(I ; \hat{q}_{\vec{x} ; r}^{1}\right)+f_{S(\vec{x} ; r)}\left(I ; \hat{q}_{\vec{x} ; r}^{2}\right)+f_{S(\vec{x} ; r)}\left(I ; \hat{q}_{\vec{x} ; r}^{3}\right)$, where

$f_{S(\vec{x} ; r)}(I ; \hat{q})=\frac{1}{4 \pi r^{2}} \int_{\partial S(\vec{x} ; r)}\left(\hat{q} \cdot \vec{\nabla} I\left(\vec{x}-r \hat{n}_{A}\right)\right)\left(\hat{q} \cdot \hat{n}_{A}\right) d A$. 
Among all possible orientations, interested detection direction is the most contributive one which yields the strongest discontinuity response magnitude, i.e.

$$
\hat{q}_{\vec{x} ; r}^{1}=\arg \max _{\hat{q}}\left|f_{S(\vec{x} ; r)}(I ; \hat{q})\right| .
$$

Considering only the most contributive orientation, the response of the intensity discontinuity descriptor $f_{S(\vec{x} ; r)}(I)$ is distilled into $f_{S(\vec{x} ; r)}\left(I ; \hat{q}_{\vec{x} ; r}^{1}\right)$. This descriptor is referred to as the distilled intensity discontinuity descriptor,

$$
f_{S(\vec{x} ; r)}^{\prime}(I)=\left\{\begin{array}{c}
\frac{1}{4 \pi r^{2}} \max _{\hat{q}}\left(\int_{\partial S_{r}}\left(\frac{\partial}{\partial \hat{q}} I\left(\vec{x}-r \hat{n}_{A}\right)\right)\left(\hat{q} \cdot \hat{n}_{A}\right) d A\right), \\
\text { if } f_{S(\vec{x} ; r)}(I)>0, \\
\quad \text { if } f_{S(\vec{x} ; r)}(I)=0, \\
\frac{1}{4 \pi r^{2}} \min _{\hat{q}}\left(\int_{\partial S_{r}}\left(\frac{\partial}{\partial \hat{q}} I\left(\vec{x}-r \hat{n}_{A}\right)\right)\left(\hat{q} \cdot \hat{n}_{A}\right) d A\right), \\
\text { if } f_{S(\vec{x} ; r)}(I)<0 .
\end{array}\right.
$$

The dot product term $\hat{q} \cdot \hat{n}_{A}$ in Equation 6 allows the distilled descriptor $f_{S(\vec{x} ; r)}\left(I ; \hat{q}_{\vec{x} ; r}^{1}\right)$ to focus on the region where $\hat{q}_{\vec{x}, r}^{1}$ is aligned along $\hat{n}_{A}$. It omits or suppresses the less contributive intensity discontinuity responses, which occur on $\partial S(\vec{x} ; r)$ with large discrepancy between $\hat{q}_{\vec{x} ; r}^{1}$ and $\hat{n}_{A}$. The less contributive responses are handled by $f_{S(\vec{x} ; r)}\left(I ; \hat{q}_{\vec{x} ; r}^{2}\right)$ and $f_{S(\vec{x} ; r)}\left(I ; \hat{q}_{\vec{x} ; r}^{3}\right)$. The distilled intensity discontinuity descriptor response computed at a position inside a non-spherical object or distant from the object boundary remains strong. It is more flexible to return responses for handling vasculatures of varying shapes and sizes than the spherical intensity discontinuity descriptor does. Based on the sign of $f_{S(r ; \vec{x})}(I)$, the strongest response is chosen as Equation 8. The offset and distilled DH ratio is formulated as,

$$
\frac{f_{S(\vec{x} ; r)}^{\prime}(I)}{\sqrt{\operatorname{Var}_{S(\vec{x}, r)}(I)}+\sqrt{\frac{3}{4 \pi r^{3}} \operatorname{Var}_{\Omega}(I)+\frac{1}{2} \rho^{2}}} .
$$

\section{Final DH Ratio}

This section revises the offset and distilled $\mathrm{DH}$ ratio and devises the corresponding analytical form for computation on discrete images. This leads to the development of the final $\mathrm{DH}$ ratio. For discrete image signals, the spherical region in the formulation of $f_{S(\vec{x} ; r)}^{\prime}$ and $\operatorname{Var}_{S(\vec{x} ; r)}$ is represented as a slightly smoothed spherical step function. It is defined as,

$$
d_{r}(\vec{x})=\left(g * d_{r}^{\prime}\right)(\vec{x}) \text { and } d_{r}^{\prime}(\vec{x})= \begin{cases}1, & \text { if }|\vec{x}| \leq r \\ 0, & \text { otherwise }\end{cases}
$$

where $g$ is Gaussian with a scale factor of 1 voxel length and $*$ is the convolution operator. Using the smoothed step function, $\operatorname{Var}_{S(\vec{x} ; r)}$ is calculated analogous to weighted local intensity variance [23]. Denote the local intensity variance computed from the smoothed step function by $w_{r}(\vec{x})$, defined as,

$$
\begin{aligned}
w_{r}(\vec{x})= & \frac{3}{4 \pi r^{3}} \int_{\Omega} I^{2}(\vec{x}+\vec{v}) d_{r}(\vec{v}) d \vec{v} \\
& -\left(\frac{3}{4 \pi r^{3}} \int_{\Omega} I(\vec{x}+\vec{v}) d_{r}(\vec{v}) d \vec{v}\right)^{2}, \\
= & \frac{12 \pi r^{3} d_{r} * I^{2}(\vec{x})-9\left(d_{r} * I(\vec{x})\right)^{2}}{16 \pi^{2} r^{6}} .
\end{aligned}
$$

The local intensity variance can be computed using the Fourier domain technique. Denote the frequency (in cycle-per-image) in the Fourier domain by $\vec{u}, \mathcal{F}$ and $\mathcal{F}^{-1}$ are respectively the fast Fourier transform and inverse fast Fourier transform operators, i.e. $\mathcal{F}\{I\}(\vec{u})$ is the Fourier coefficient of $I$ at the frequency $\vec{u}$ and $\mathcal{F}^{-1}\{\mathcal{F}\{I\}\}(\vec{x}) \equiv I(\vec{x})$. Equation 11 is evaluated in the Fourier domain, as stated in Equation 12.

$$
\begin{aligned}
& w_{r}(\vec{x}) \\
& =\frac{12 \pi r^{3} \mathcal{F}^{-1}\left\{\mathcal{F}\left\{d_{r}\right\}(\vec{u}) \mathcal{F}\left\{I^{2}\right\}(\vec{u})\right\}(\vec{x})-9\left(\mathcal{F}^{-1}\left\{\mathcal{F}\left\{d_{r}\right\}(\vec{u}) \mathcal{F}\{I\}(\vec{u})\right\}(\vec{x})\right)^{2}}{16 \pi^{2} r^{6}},
\end{aligned}
$$

where by the Hankel transform [24], $\mathcal{F}\left\{d_{r}\right\}(\vec{u})=$ $e^{-2 \pi^{2}|\vec{u}|^{2}} \frac{2 r^{3} \sin (2 r \pi|\vec{u}|)-4 r^{4}|\vec{u}| \cos (2 r \pi|\vec{u}|)}{3 \pi|\vec{u}|^{3}}$.

Along the same line, the distilled intensity discontinuity descriptor is reformulated using the smoothed step function (Appendix B). Based on this reformulation, the distilled intensity discontinuity descriptor is computed as the eigenvalues of a 3-by-3 tensor. The eigenvalues of the tensor are represented as $\lambda \frac{1}{x}, r, \lambda_{\vec{x}, r}^{2}$ and $\lambda_{\vec{x}, r}^{3}$, where $\lambda_{\vec{x}, r}^{1} \leq \lambda_{\vec{x}, r}^{2} \leq \lambda_{\vec{x}, r}^{3}$. The terms $\lambda_{\vec{x}, r}^{3}$ and $\lambda_{\vec{x}, r}^{1}$ correspond to the resultant values of the case of $f_{S(\vec{x} ; r)}(I)>0$ and the case of $f_{S(\vec{x} ; r)}(I)<0$ (the first and the third rows) in Equation 8 respectively. Equation 8 becomes,

$$
h_{r}(\vec{x})= \begin{cases}\lambda_{\vec{x}, r}^{3}, & \text { if } \lambda_{\vec{x}, r}^{1}+\lambda_{\vec{x}, r}^{2}+\lambda_{\vec{x}, r}^{3}>0, \\ 0, & \text { if } \lambda_{\vec{x}, r}^{1}+\lambda_{\vec{x}, r}^{2}+\lambda_{\vec{x}, r}^{3}=0, \\ \lambda \vec{x}, r^{1}, & \text { if } \lambda_{\vec{x}, r}^{1}+\lambda_{\vec{x}, r}^{2}+\lambda_{\vec{x}, r}^{3}<0 .\end{cases}
$$

Finally, the offset and distilled DH ratio, with the smoothed local spherical region (so as the Fourier domain implementation), is

$$
R_{r}(\vec{x})=\frac{h_{r}(\vec{x})}{\sqrt{w_{r}(\vec{x})}+\sqrt{\frac{3}{4 \pi r} \operatorname{Var}_{\Omega}(I)+\frac{1}{2} \rho^{2}}} .
$$

The intensity based image features and the associated DH ratios are summarized in Table I.

\section{LeVel Set BASed Deformable Models}

This section elaborates three active contour models. The first model devised in Section III-A utilizes the lowest complexity version of the proposed descriptor - the spherical intensity discontinuity descriptor. It offers a preliminary segmentation of the target vasculature in order to estimate the parameter $\rho$ for the final DH ratio. Based on the estimated parameter, the second model presented in Section III-B segments the normal vessels and most of the aneurysmal regions using the final $\mathrm{DH}$ ratio. Inside aneurysm domes, where severe turbulent flows happen, regions missed by the DH ratio based active contour model are handled by the third deformable model. This procedure is referred to as topology refinement and is elaborated in Section III-D.

\section{A. Preliminary Segmentation and Estimation of Parameter $\rho$}

The preliminary segmentation extracts the major vessels in order to estimate the parameter $\rho$ for handling the external bias which adversely affects the $\mathrm{DH}$ ratio based detection. The spherical intensity discontinuity descriptor (Equation 1) 
TABLE I

Summary of the INTENSITy Based IMAGe Features AND the Associated DH RATIOS

\begin{tabular}{|c|c|c|c|c|c|c|}
\hline \multicolumn{3}{|c|}{ Intensity based image features } & \multicolumn{3}{|c|}{ Ratios derived from the intensity based image features } & \\
\hline $\begin{array}{c}\text { Spherical } \\
\text { intensity } \\
\text { discontinuity } \\
\text { descriptor }\end{array}$ & $\begin{array}{c}\text { Distilled } \\
\text { intensity } \\
\text { discontinuity } \\
\text { descriptor }\end{array}$ & $\begin{array}{c}\text { Local } \\
\text { intensity } \\
\text { variance }\end{array}$ & Offset $\mathrm{DH}$ ratio & $\begin{array}{c}\text { Offset and distilled } \\
\text { DH ratio }\end{array}$ & Final DH ratio & \\
\hline \multirow[t]{2}{*}{ (1) } & (7) & (11) & (4) & (9) & (14) & \\
\hline & & $\checkmark$ & $\checkmark$ & $\checkmark$ & $\checkmark$ & $\begin{array}{c}\text { Detect intensity changes } \\
\text { inside } S(\vec{x}, r)\end{array}$ \\
\hline \multirow[t]{4}{*}{$\checkmark$} & $\checkmark$ & & $\checkmark$ & $\checkmark$ & $\checkmark$ & $\begin{array}{c}\text { Detect intensity changes } \\
\text { at } \partial S(\vec{x}, r)\end{array}$ \\
\hline & & & $\checkmark$ & $\checkmark$ & $\checkmark$ & $\begin{array}{l}\text { Avoid small variances } \\
\text { exaggerating the ratio }\end{array}$ \\
\hline & $\checkmark$ & & & $\checkmark$ & $\checkmark$ & $\begin{array}{l}\text { Less restrictive detection } \\
\text { for non-spherical objects }\end{array}$ \\
\hline & & & & & $\checkmark$ & $\begin{array}{l}\text { Analytical formulation for } \\
\text { discrete images }\end{array}$ \\
\hline
\end{tabular}

resembles the normalized discrete Laplacian operator proposed in Flux maximizing geometric flows [16], [17]. Although it is possibly unsuitable to segment low contrast aneurysms (discussed in Section IV-B), it well extracts strong intensity major vessels. The preliminary segmentation follows the fluxbased contour evolution equation described in [16], [17],

$$
\begin{gathered}
\left.\frac{d \psi_{\mathrm{FLUX}}}{d t}\right|_{\psi_{\mathrm{FLUX}}=0}=f_{S\left(\vec{x} ; r_{\mathrm{FLUX}}\right)}^{\prime}(I)\left|\vec{\nabla} \psi_{\mathrm{FLUX}}\right| \\
+\kappa^{\prime} \vec{\nabla} \cdot \frac{\vec{\nabla} \psi_{\mathrm{FLUX}}}{\left|\vec{\nabla} \psi_{\mathrm{FLUX}}\right|}, \\
\text { where } r_{\mathrm{FLUX}}=\arg \max _{r \in \mathbf{R}}\left|f_{S(\vec{x} ; r(\vec{x}))}^{\prime}(I)\right|,
\end{gathered}
$$

where $\psi_{\text {Fux }}$ is a level set function, of which the zero level surface represents the evolving surface [25]. The value of $\kappa^{\prime}$ governs the smoothness of the resultant contour. It determines the strength of the curvature regularization force which restrains the contours from overshooting the structure boundary. This parameter is 0.2 in all the experiments. The initial level set function for preliminary segmentation is acquired by thresholding the voxels exhibiting the highest $0.01 \%$ intensity among all voxels. Other parameters to solve the above differential equation are set according to the descriptions in [26] and the implementation is grounded on [27]. The evolution of the level set function is stopped when the accumulated per-voxel update of the level set function is less than $10^{-5}$ for 10 iterations. Subsequent to the preliminary segmentation, the value of $\rho$ is estimated as the difference between the averaged intensity inside the segmented region, and the averaged intensity of the region within a distance of 2 voxel-length outside the segmented region. This estimation returns a value which roughly represents the intensity contrast between the major vessels and their vicinity.

\section{B. Active Contour Model Based on the Final DH Ratio}

Denote the enclosed evolving surface by $\mathcal{C}$, the resultant surface of this active contour model is found by maximizing an energy functional $E(\mathcal{C})$, i.e. $\arg \max _{\mathcal{C}}(E(\mathcal{C}))$ and

$$
E(\mathcal{C})=-\int_{\text {Volume }(\mathcal{C})} R_{r(\mathcal{C}(\vec{s}))}(\mathcal{C}(\vec{s})) d \vec{s}-\kappa \int_{\operatorname{Area}(\mathcal{C})} d \vec{w},
$$

where $\kappa$ specifies the strength of the surface smoothness constraint, $r(\mathcal{C}(\vec{s}))$ is the desired detection range at the position $\mathcal{C}(\vec{s})$ and is discussed in the next section, $\vec{s}$ and $\vec{w}$ are respectively the parameterization of the enclosed volume of $\mathcal{C}$ and the surface of $\mathcal{C}$. Denote the level set function is $\psi(\vec{x})$ which represent $\mathcal{C}$, the dynamic of the level set function is,

$$
\left.\frac{d \psi(\vec{x})}{d t}\right|_{\psi=0}=R_{r(\vec{x})}(\vec{x})|\vec{\nabla} \psi(\vec{x})|+\kappa\left(\vec{\nabla} \cdot \frac{\vec{\nabla} \psi(\vec{x})}{|\vec{\nabla} \psi(\vec{x})|}\right) .
$$

$\kappa$ is 0.05 in all experiments to maintain surface smoothness. The evolving contour is expanded by positive values $R_{r(\vec{x})}(\vec{x})$ inside strong intensity vasculatures. It is negative in the vicinity of these vasculatures and in the regions affected by severe turbulent flows, where the contour is shrunk to avoid leakages. Owing to the surged local intensity variance, the magnitude of $R_{r(\vec{x})}(\vec{x})$ is minor at vessel boundaries. In this situation, the dynamic of the evolving contour is mainly governed by the smoothness term.

\section{Detection Range Selection}

In the computation of $R_{r(\vec{x})}(\vec{x})$, the value of $r(\vec{x})$ specifies the size of the local spherical region. A proper value is the distance from the local voxel to the closest object boundary. It allows the sphere surface to reach the object boundary to induce a significant detection response in order to reliably guide the evolving surface.

An undersized $r$ disallows the sphere surface touching the object boundary, in which the computation result of the intensity discontinuity descriptor and the DH ratio are unreliable. Meanwhile, if the sphere overshoots the object boundary, a large local intensity variance is given, and consequently penalizes the ratio based measure response. The radius that gives the largest negative final $\mathrm{DH}$ ratio obtained among a 


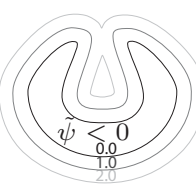

(a)

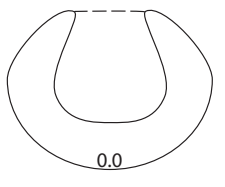

(b)

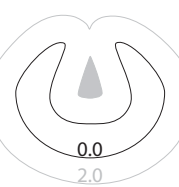

(c)

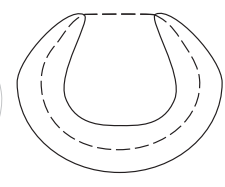

(d)
Fig. 2. For illustration only, an 2-D concave-cavity example. (a)-(c) Numbers represent the contour isovalues of the function $\tilde{\psi}(\vec{x})$, which is the signed distance function of the $\mathrm{DH}$ ratio based active contour segmentation result [i.e. evolution result of (17)]. (b) Dotted line is the valley of $\tilde{\psi}(\vec{x})$ separating the concave-concavity and the background. (c) Shaded region is the marker found by performing erosion and connectivity analysis on $\tilde{\psi}(\vec{x})$, i.e., $\bigcup_{i \neq q_{2}} \mathbf{C}_{i, \tilde{\psi}(\vec{x})>2}$, where $q_{2}=\arg \max _{i}\left|\mathbf{C}_{i, \tilde{\psi}(\vec{x})>2}\right|$. (d) Dotted line is the contour of the reclaimed concave-cavity by expanding the marker shown in (c) along with the level set evolution function (19).

predefined detection range set $\mathbf{R}$ is chosen. This set comprises the distances ranged from the smallest voxel-length to the size of the largest part of the target structure (the size of the aneurysm). This allows $R_{r(\vec{x})}(\vec{x})$ to convey negative responses to expand contours inside the entire vasculature. Meanwhile, the selected range should also deliver positive detection response in the smallest detection range to shrink the evolving contour when it starts overshooting the structure boundaries. Therefore,

$$
\begin{aligned}
& \qquad r(\vec{x})=\left\{\begin{array}{cc}
l, & \text { if } R_{l}(\vec{x})>\left|R_{r^{-}(\vec{x})}(\vec{x})\right|, \\
r^{-}(\vec{x}), & \text { otherwise. }
\end{array} \text { where } \quad r^{-}(\vec{x})=\arg \max _{r \in \mathbf{R}}\left|\min \left(R_{r}(\vec{x}), 0\right)\right| .\right.
\end{aligned}
$$

The detection range set $\mathbf{R}$ contains the distances ranged from the smallest voxel-length (denoted as $l$ ) to at least the size of the aneurysm for every voxel-length. The final $\mathrm{DH}$ ratio computed from oversize ranges is penalized by a large local intensity variance, the range set $\mathbf{R}$ can contain values larger than the actual size of the desired objects. In practice, it is possible to make use of a large detection range set to cope with different images with structures having various sizes.

\section{Topology Refinement}

Inside an aneurysm domes, the blood flows in a vortex-like pattern [10], [28], [29]. It sharply reduces the flow-sensitive voxel intensity inside a dome, particularly around the center of the turbulent flow. As a result, the segmentation scheme using the intensity based features misses the interior of the dome. This creates unsegmented regions in aneurysm domes and either becomes isolated unsegmented regions (referred to as holes hereafter), or create unsegmented concave-cavities in the intensity feature-based segmentation result. A hole is a group of voxels which are disconnected from the background region. A concave-cavity is connected with the background region, through one or more openings on the segmented structure surrounding that concave-cavity. The widths of the openings are relatively small compared to the minimum width of the corresponding concave-cavity.

The major intracranial arteries captured by PC-MRA images are rarely closely packed or twisted to entirely enclose a small region to form an isolated (a hole) or almost-isolated (a concave-cavity) background region. Hence, by identifying

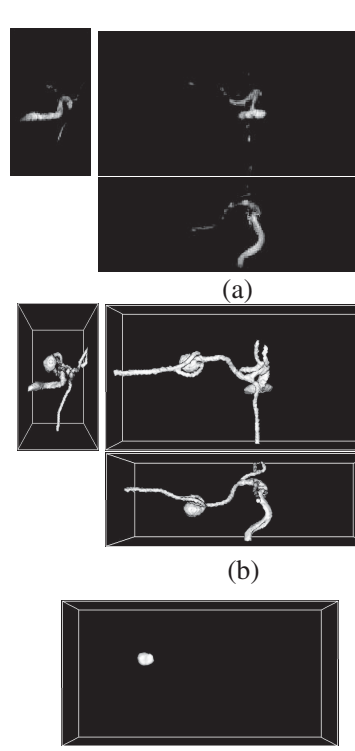

(c) (d)

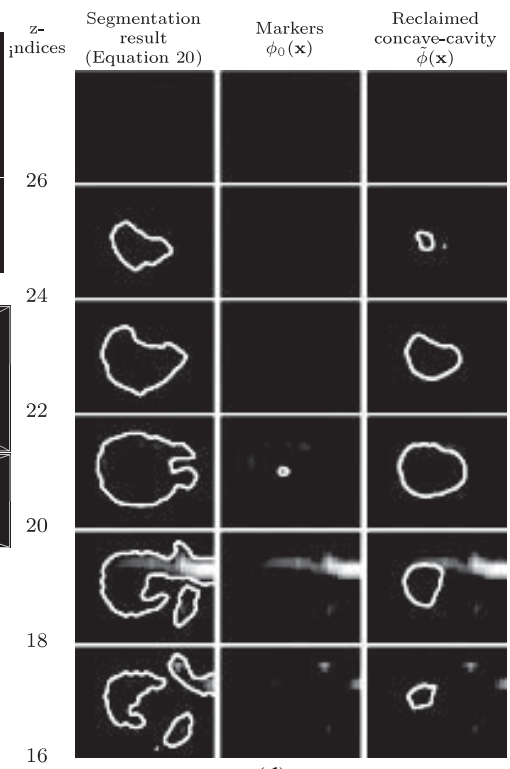

Fig. 3. Experiment on the vascular phantom. (a) Perspective maximum intensity projection images of the phantom image volume (voxel size: $0.86 \times$ $0.86 \times 1.00 \mathrm{~mm}^{3}$, image dimension: $100 \times 192 \times 56$ ). (b) Segmentation result. (c) Reclaimed holes and concave-cavities, which also suggest the aneurysm position. (d) Zoomed-in image of the aneurysmal region. The segmentation result is obtained by using $\mathbf{R}=\{0.86,1.72,2.58,3.44,4.30,5.16\} \mathrm{mm}, \mathbf{D}=$ $\{0.43,0.86,1.29,1.72,2.15,2.58,3.01,3.44\} \mathrm{mm}$. The initial seed points of the level set function $\psi$ are obtained by thresholding the brightest $0.1 \%$ voxels.

the holes or the concave-cavities, the algorithm can reclaim the regions where the flow-sensitive intensity is severely reduced by the turbulence flows and undetected by the $\mathrm{DH}$ ratio based segmentation scheme.

Denote the resultant level set function of the DH ratio based segmentation scheme (i.e. the evolution result of Equation $17)$ is $\tilde{\psi}(\vec{x})$, the segmented and unsegmented structures are respectively the regions $\{\tilde{\psi}(\vec{x})<0\}$ and $\{\tilde{\psi}(\vec{x}) \geq 0\}$ (see Fig. 2(a)). Adjacent voxels are regarded as connected if they belong to the same region type in the 26-neighborhood system. By performing connectivity analysis, we define $\mathbf{C}_{i, \tilde{\psi}(\vec{x})>0}$ as the set of the voxels of the $i$ th connected component of the region $\{\tilde{\psi}(\vec{x})>0\}$. The holes are found as the unsegmented voxels, which are disconnected from background (the unsegmented component of the largest number of voxels), i.e.

$$
\bigcup_{i \neq q} \mathbf{C}_{i, \tilde{\psi}(\vec{x})>0},
$$

where $q=\arg \max _{i}\left|\mathbf{C}_{i, \tilde{\psi}(\vec{x})>0}\right|$.

A concave-cavity is defined as a group of unsegmented voxels, surrounded by a segmented structure with one or more small openings on that surrounding structure. The openings on that structure are a few low intensity voxels located at the outer parts of the aneurysm having fluctuating intensity. The width of an opening caused by voxel intensity fluctuation is assumed to be smaller than that of the concave-cavity corresponding to the vortex-like turbulent flow center.

For illustration only, Fig. 2 presents a 2D example of a concave-cavity. The concave-cavity connects with the background through a relatively small opening (the dotted line in 


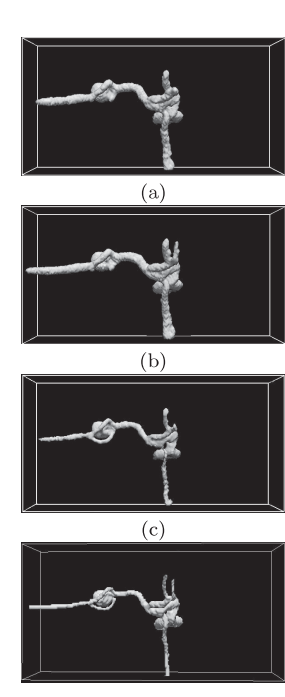

(d)

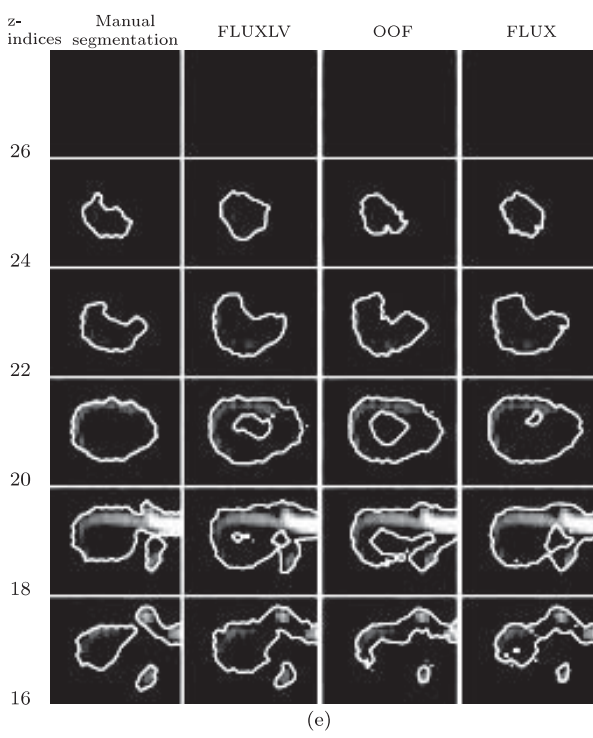

(e)

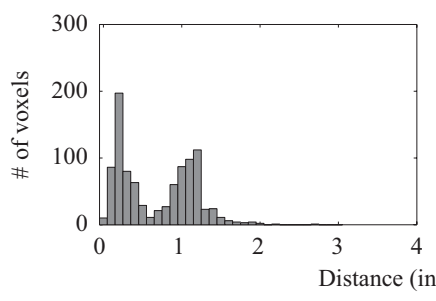

(a)

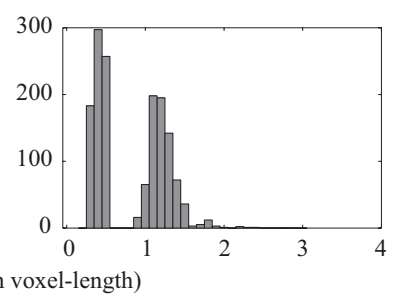

(b)

Fig. 6. Histograms of (a) distances from the false negative voxels to the closest segmented region boundary and (b) false positive voxels to the closest ground truth boundary.

The recovery of the concave-cavities is accomplished by using a two stage procedure. In the first stage, markers are identified in each concave-cavity by performing connectivity analysis on a set of eroded unsegmented regions, $\bigcup_{d \in \mathbf{D}} \bigcup_{i \neq q_{d}} \mathbf{C}_{i, \tilde{\psi}(\vec{x})>d}$, where $q_{d}=\arg \max _{i}\left|\mathbf{C}_{i, \tilde{\psi}(\vec{x})>d}\right|$ and $\mathbf{D}$ is a set of erosion distances. Given an erosion distance $d$ is larger than the widths of the openings but smaller than the associated concave-cavities, the small openings between sealed (see the example in Fig. 2(c)). A sealed concavecavity becomes an unsegmented component isolated from the unsegmented background and thus, can be identified by the connectivity analysis.

The erosion distance set $\mathbf{D}$ is different from the previously introduced detection range set $\mathbf{R}$. The best choice of the distances in $\mathbf{D}$ contains only the widths of the openings which connect the unsegmented concave-cavities to the unsegmented background. It ensures that the concave-cavities are properly recognized, and simultaneously avoids the background being surrounded by multiple vessel branches from being misclassified as a concave-cavity. In Fig. 2(c), a marker is identified by finding the isolated region of $\{\tilde{\psi}>2\}$. In practice, we suggest employing distances ranged from 0.5 voxel-length, in every 0.5 voxel-length, to the radius of the major vessels.

In the second stage, the markers are evolved as deformable surfaces. The surfaces expand until they reach their surrounding valleys of $\tilde{\psi}(\vec{x})$. It is achieved by evolving another level set function $\phi(\vec{x})$. The zero level of this function represents the boundaries of the deforming surfaces of the reclaimed regions. The initial level set function (denoted as $\phi_{0}(\vec{x})$ ) is acquired by assigning -1 to the positions of the markers and 1 to the background. The implementation of the evolution of this level set function is the same as that of the previous one. The dynamic of $\phi(\vec{x})$ is described by

$$
\left.\frac{\partial \phi(\vec{x})}{\partial t}\right|_{\phi=0}=\vec{\nabla} \tilde{\psi}(\vec{x}) \cdot \vec{\nabla} \phi(\vec{x}) .
$$

Fig. 5. Experiment on the vascular phantom. The images depicted are zoomed-in images of the segmentation results of different methods based on the phantom image volume (Fig. 3) before topology refinement is performed.

Fig. 2(b)) on the surrounding segmented region. Inside the concave-cavity, there are some positions where the values of the signed distance function $\tilde{\psi}(\vec{x})$ are larger than those values at all the positions on the small opening (see Fig. 2(a)). Across the small opening, a local valley on the function $\tilde{\psi}(\vec{x})$ is observed. This local valley is one of the features found across the openings associated with concave-cavities.

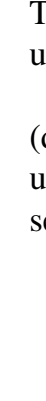
up the unsegmented concave-cavity (see Fig. 2(d)).

Finally, the identified holes and filled concave-cavities (denoted as $\{\tilde{\phi}(\vec{x})<0\}$ ), together with the segmented region using intensity based features $\{\tilde{\psi}(\vec{x})<0\}$, are the final segmented vasculature,

$$
\bigcup\left\{\{\tilde{\phi}(\vec{x})<0\},\{\tilde{\psi}(\vec{x})<0\}, \bigcup_{i \neq q} \mathbf{C}_{i, \tilde{\psi}(\vec{x})>0}\right\} .
$$


FLUXLV

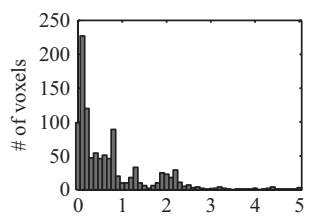

(a)
OOF

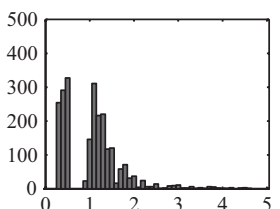

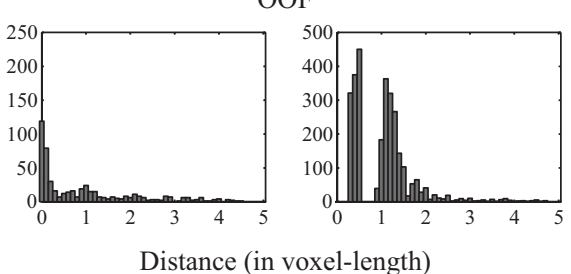

(b)
FLUX
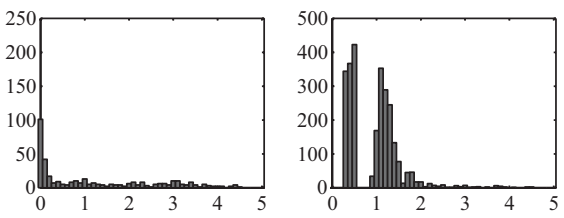

(c)

Fig. 7. (a)-(c) Histograms of the distances from the false negative voxels to the closest segmented region boundary (left in each subfigure) and the false positive voxels to the closest ground truth boundary (right in each subfigure).

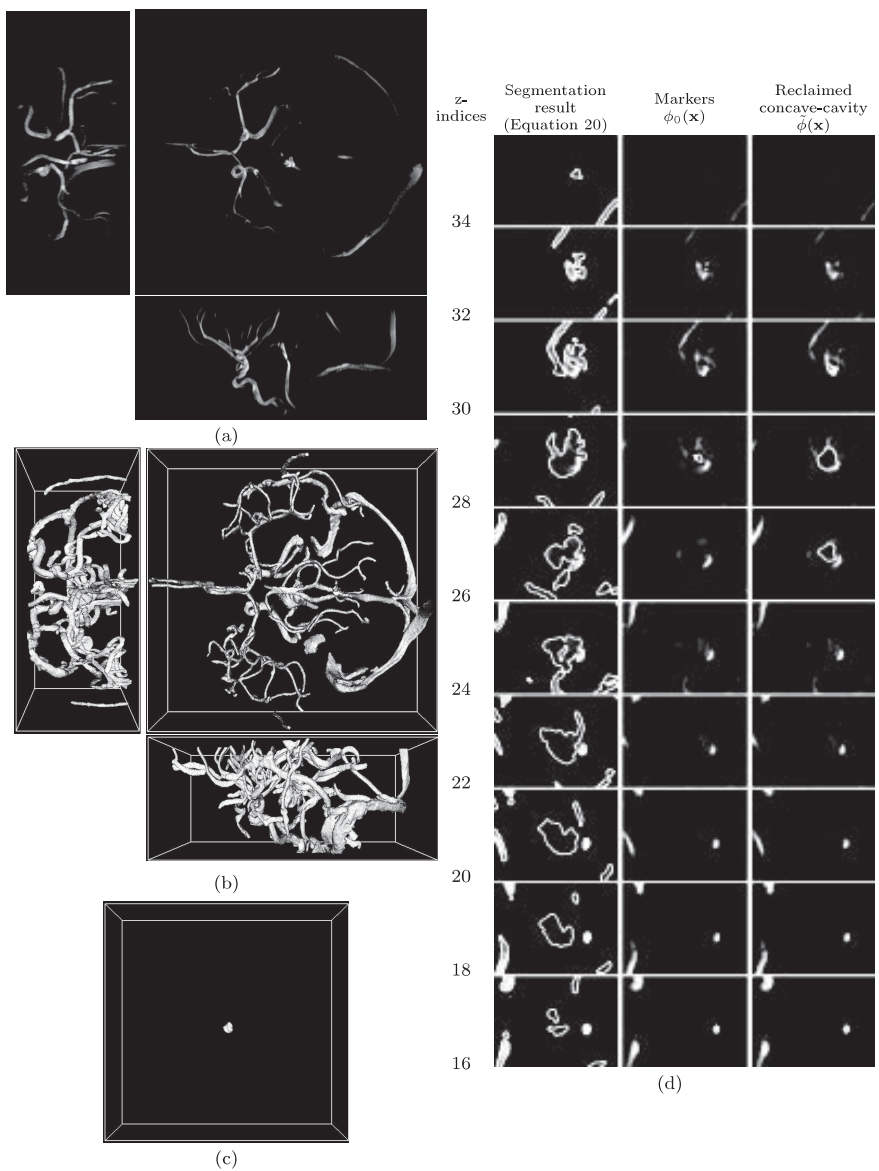

Fig. 8. Experiment on the first clinical case, an aneurysm at the basilar artery. (a) PC-MRA image volume (voxel size: $0.4 \times 0.4 \times$ $1.0 \mathrm{~mm}^{3}$, image dimension: $386 \times 377 \times 66$ voxels $)$ used in the clinical data experiment. First row: sagittal and axial projections; second row: coronal projection. (b) Segmentation result. (c) Reclaimed holes and concave-cavities, which also suggest the aneurysm position. (d) Zoomed-in images of the aneurysmal region. The segmentation result is obtained by using $R=\{0.4,0.8,1.2,1.6,2.0,2.4,2.8,3.2\} \mathrm{mm}, D=$ $\{0.2,0.4,0.6,0.8,1.0,1.2,1.4,1.6,1.8,2.0,2.2,2.4,2.6,2.8,3.0,3.2\} \mathrm{mm}$. The initial seed points of the level set function $1 \%$ are obtained by thresholding the brightest $1 \%$ voxels.

This final segmented region consists of both the normal vessels and the aneurysmal region.

\section{EXPERIMENT}

\section{A. Experiment Settings}

The proposed method has been evaluated on 5 PC-MRA volumetric images. The first image volume has a large aneurysm which was scanned using a PC-MRA protocol on a 1.5T GE MR scanner at the Department of Neuroscience, King's College London, London. The rest of the volumes are clinical PC-MRA images, which were acquired on a Philips 3T ACS Gyroscan MR scanner at the University Hospital of Zurich. The image intensity was scaled to the range within $[0,1]$. Through visual inspection, the parameters of the proposed method - the detection range set $\mathbf{R}$ and erosion distance set $\mathbf{D}$ were specified for each case. As mentioned in Sections III-B and C, $\mathbf{R}$ and $\mathbf{D}$ are assigned with the consideration of the smallest voxel-length, the size of the aneurysm and the radius of the major vessels. The largest values in $\mathbf{R}$ and $\mathbf{D}$ are defined slightly larger than the actual sizes of all target vessels to ensure these structures are properly considered. The level set function $\psi$ is initialized by selecting a proportion of voxels with the highest intensity. The proportion is manually specified to each case to ensure that there is at least one seed point in each component of the desired vascular structures, while keeping all seed points out of the image background.

\section{B. Methods for Comparison}

In the experiments, the segmentation performance is studied using the same segmentation framework (with and without topology refinement), but utilizing three lower complexity variants of the proposed final DH ratio. Prior to topology refinement, these variants are closely related to three existing approaches. Thus, this performance study also reveals the advantageous performance of the proposed method over three existing intensity feature-based segmentation approaches. Based on the notation shown in Table I, these three variants are,

1) Spherical intensity discontinuity descriptor (the first column of Table I). The segmentation method based on this descriptor is the same procedure as described in Equation 15, so as Flux maximizing geometric flows [16] (FLUX).

2) Offset DH ratio (the fourth column of Table I). The offset DH ratio segmentation is closely related to our previous work [15]. It can be regarded as combining Flux maximizing geometric flows [16] and local intensity variances (FLUXLV).

3) Distilled intensity discontinuity descriptor (the second column of Table I). As discussed in Section II-C and Appendix B, this discontinuity descriptor is tangential to estimating the oriented flux along the optimal direction, as described in optimally oriented flux [30] (OOF). 
TABLE II

Segmentation Result Evaluation of the Proposed Segmentation Method for the Vascular Phantom Experiment

\begin{tabular}{|c|c|c|c|c|}
\hline & \multicolumn{2}{|c|}{ Phantom } & \\
\hline & & Vessel & Background & \\
\hline \multirow{3}{*}{$\begin{array}{l}\text { Segmentation } \\
\text { result }\end{array}$} & $\begin{array}{l}\text { Segmented } \\
\text { voxels }\end{array}$ & $\begin{array}{c}\text { True positive (TP) } \\
4913\end{array}$ & $\begin{array}{c}\text { False positive (FP) } \\
1489\end{array}$ & $\begin{array}{c}\text { Positive predictive value } \\
\frac{\mathrm{TP}}{\mathrm{TP}+\mathrm{EP}}=76.74 \%\end{array}$ \\
\hline & $\begin{array}{c}\text { Unsegmented } \\
\text { voxels }\end{array}$ & $\begin{array}{c}\text { False negative (FN) } \\
960\end{array}$ & $\begin{array}{c}\text { True negative }(\mathrm{TN}) \\
1067838\end{array}$ & $\begin{array}{c}\text { Negative predictive value } \\
\text { TN } \\
\text { TN+FN }=99.91 \%\end{array}$ \\
\hline & & $\begin{array}{c}\text { Sensitivity } \\
\frac{\mathrm{TP}}{\mathrm{TP}+\mathrm{FN}}=83.65 \%\end{array}$ & $\begin{array}{c}\text { Specificity } \\
\frac{\mathrm{TN}}{\mathrm{FP}+\mathrm{TN}}=99.86 \%\end{array}$ & $\begin{array}{c}\text { Dice coefficient [31] } \\
\frac{2 \mathrm{TP}}{2 \mathrm{TP}+\mathrm{FN}+\mathrm{FP}}=0.8004\end{array}$ \\
\hline
\end{tabular}

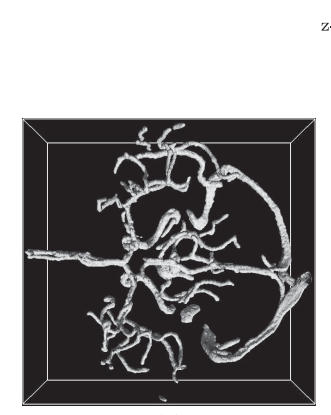

(a)

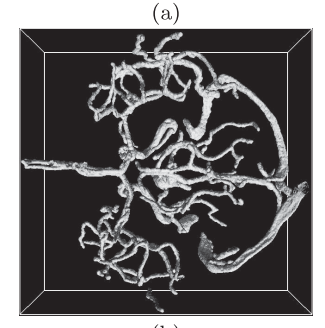

(b)

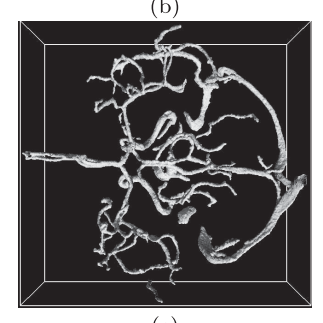

(c)

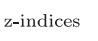

32

\section{0}

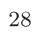

26
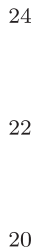

18

16
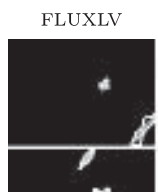

22

OOF

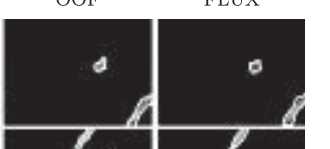

3.

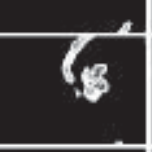

s

88

22

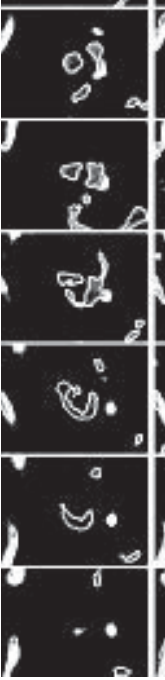

(d)

Fig. 9. Experiment on the first clinical case. The final segmentation results (20) of different methods based on the image volume [Fig. 8(a)] are depicted: (a) FLUXLV; (b) OOF; and (c) FLUX. (d) Zoomed-in images of the aneurysmal region of various methods, topology refinement cannot segment additional region for FLUXLV, OOF and FLUX in this case.

The level set evolution equation for FLUXLV ( $\psi_{\text {FLUXLV }}$ ) is obtained by replacing the term $R_{r}(\vec{x})$ in Equations 17 and 18 using the offset DH ratio (see Equation 4). The corresponding offset parameters and the curvature regularization parameter follow those used by the proposed method. Analogously, the OOF level set evolution equations $\left(\psi_{\mathrm{OOF}}\right)$ is acquired as replacing the spherical intensity discontinuity descriptor $f_{S\left(\vec{x} ; r_{\mathrm{OOF}}(\vec{x})\right)}^{\prime}(I)$ in Equation 15 using the distilled discontinuity descriptor $f_{S\left(\vec{x} ; r_{\mathrm{OOF}}(\vec{x})\right)}(I)$. According to our experiments, $\kappa^{\prime}=$ 0.2 segment the most vascular regions by OOF and FLUX
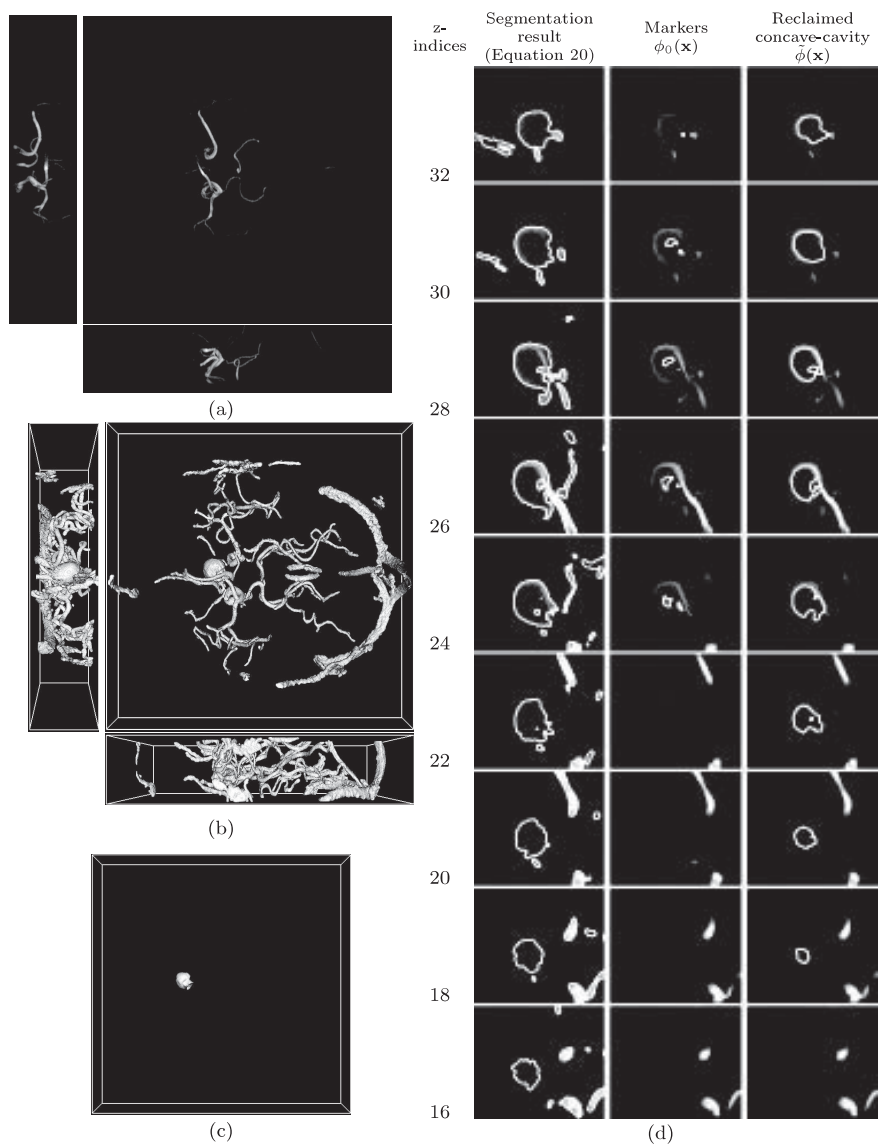

Fig. 10. Experiment on the second clinical case, an aneurysm at the anterior cerebral artery-anterior communicating artery (ACA-ACoA) junction. (a) PC-MRA image volume (voxel size: $0.4 \times 0.4 \times 1.0 \mathrm{~mm}^{3}$, image dimension: $512 \times 512 \times 45$ voxels) used in the clinical data experiment. First row: sagittal and axial projections; second row: coronal projection. (b) Segmentation result. (c) Reclaimed holes and concavecavities, which also suggest the aneurysm position. (d) Zoomed-in images of the aneurysmal region. The segmentation result is obtained by using $R=\{0.4,0.8,1.2,1.6,2.0,2.4,2.8,3.2\} \mathrm{mm}, D=\{0.2,0.4$, $0.6,0.8,1.0,1.2,1.4,1.6,1.8,2.0,2.2,2.4,2.6,2.8,3.0,3.2\} \quad \mathrm{mm}$ and $\rho=0.0272$. The initial seed points of the level set function $0.5 \%$ are obtained by thresholding the brightest $0.5 \%$ voxels.

without observable leakage among the results obtained in a set of values, ranged from 0 to 1 in a 0.05 interval. Topology refinement is applied on the level set evolution results of $\tilde{\psi}_{\mathrm{FLUXIV}}, \tilde{\psi}_{\mathrm{ooF}}$ and $\tilde{\psi}_{\mathrm{FLUX}}$, similar to the proposed method. For those evolution results where neither concave-cavity nor hole is found, the evolution results and the corresponding topology refinement results are identical. The latter is therefore not 


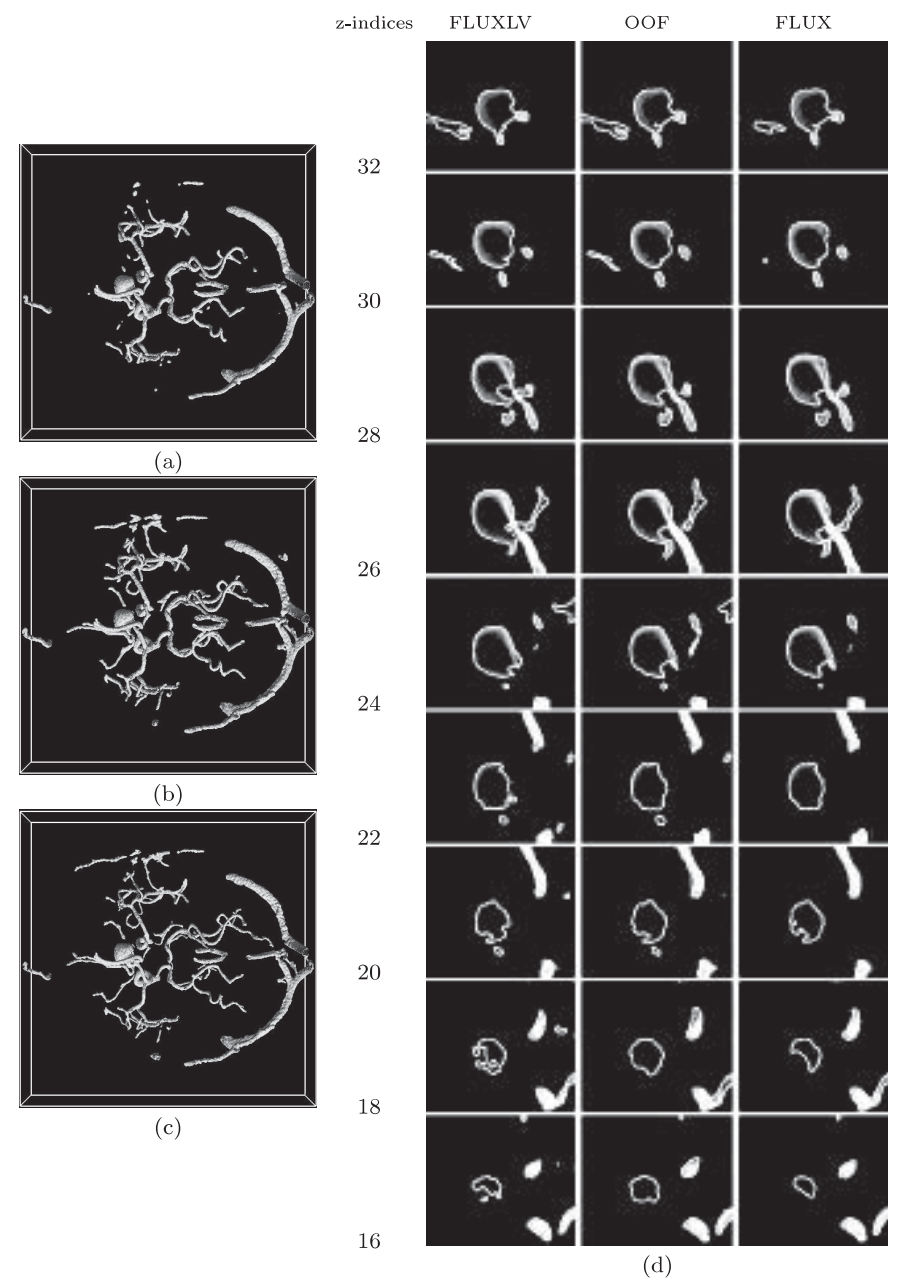

Fig. 11. Experiment on the second clinical case. The final segmentation results (20) of different methods based on the image volume [Fig 10(a)] are depicted: (a) FLUXLV; (b) OOF; and (c) FLUX. (d) Zoomed-in images of the aneurysmal region of various methods.

presented in this paper. For each individual case, the detection range set $\mathbf{R}$, erosion distance set $\mathbf{D}$ and initial contours of all methods are the same.

\section{Experimental Results}

In the first experiment, the image volume with a large aneurysm was employed (Fig. 3(a)). The segmentation result of the proposed method is shown in Fig. 3(b). The zoomin image slices are displayed in Fig. 3(d), where the final segmentation result, markers, reclaimed holes and concavecavities are presented together. The position of the aneurysm is indicated as the reclaimed hole and concave-cavity in Fig. 3(c). The final DH ratio helps the contour propagate from the vessel to the aneurysmal region. Inside the aneurysm dome, where the turbulent flow sharply reduces the voxel intensity, the topology refinement scheme recovers the region which is undetected by the DH ratio based active contour model. The proposed method gives a promising segmentation result (see Table II) as compared to the ground truth segmentation. The corresponding Dice similarity measure is 0.8004 , which is the best among 0.7439 (FLUXLV), 0.7843 (OOF) and 0.7578 (FLUX) as shown in the lower right entry of each corresponding sub-table

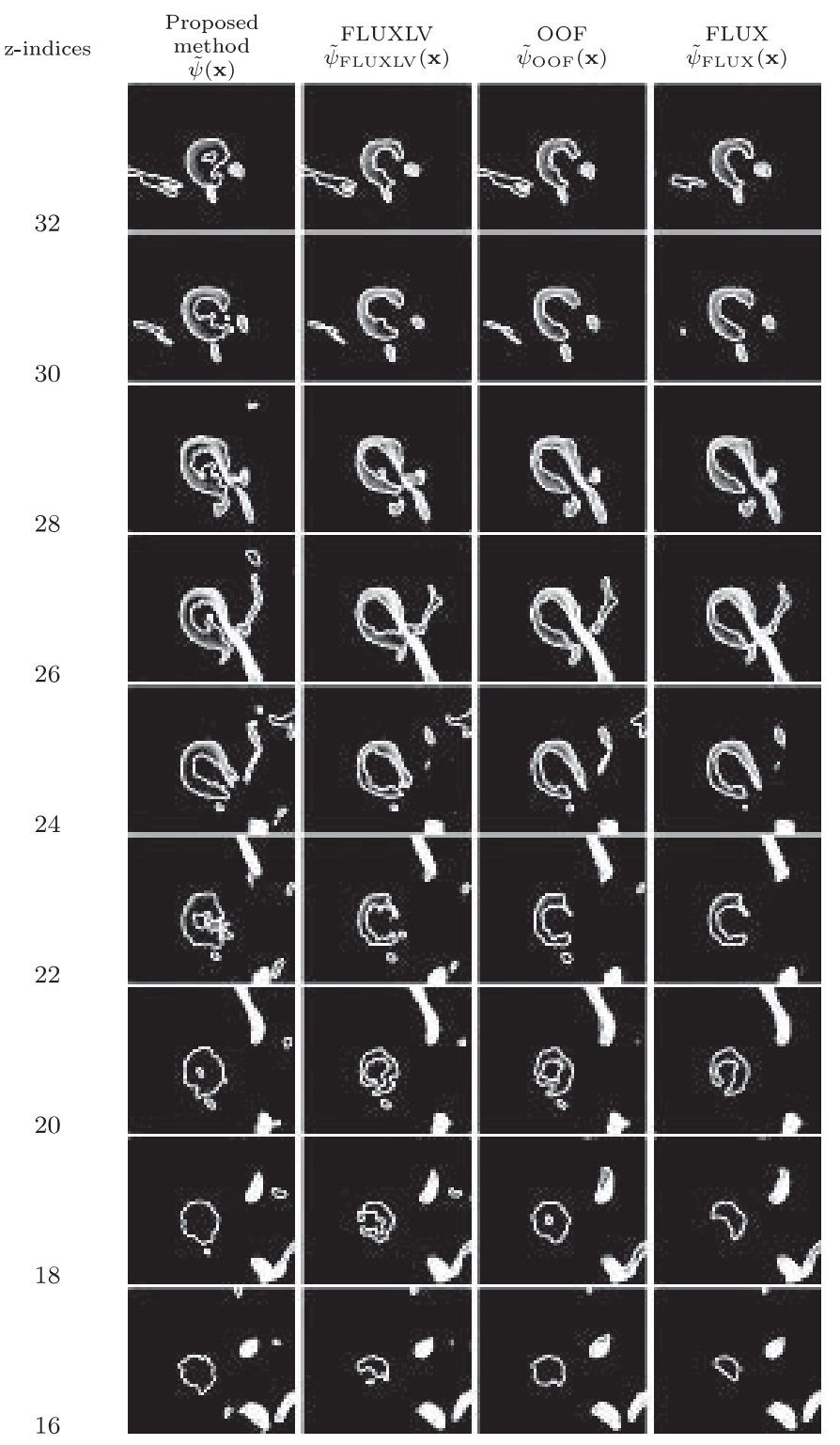

Fig. 12. Experiment on the second clinical case. The images depicted are zoomed-in images of the segmentation results of different methods based on the clinical PCMRA image volume [Fig. 10(a)], before topology refinement is performed.

in Table III. Compared to the results obtained by FLUXLV, OOF and FLUX (see Figs. 4(a)-(c)), the segmentation result of the proposed method (Fig. 3(b)) is the closest to the manually segmented vasculature shown in Fig. 4(d). Before the topology refinement step, the proposed method can generally capture more aneurysmal region than FLUXLV, OOF and FLUX do (compare the first column against the second to the fourth columns in Fig. 5). As such, after the topology refinement step, the segmented aneurysmal region of the proposed method (the first column of Fig. 3(d)) gives the best match to the manual segmentation (the first column of Fig. 5) as compared to FLUXLV, OOF and FLUX (the second to fourth columns of Fig. 5)).

Segmentation error is further evaluated by investigating the distance from the false negative voxels to the closest segmented region boundary, and the distance from the false positive voxels to the closest ground truth vessel boundary. 
TABLE III

Segmentation Result Evaluation of FLUXLV, OOF, and FLUX for the Vascular Phantom Experiment

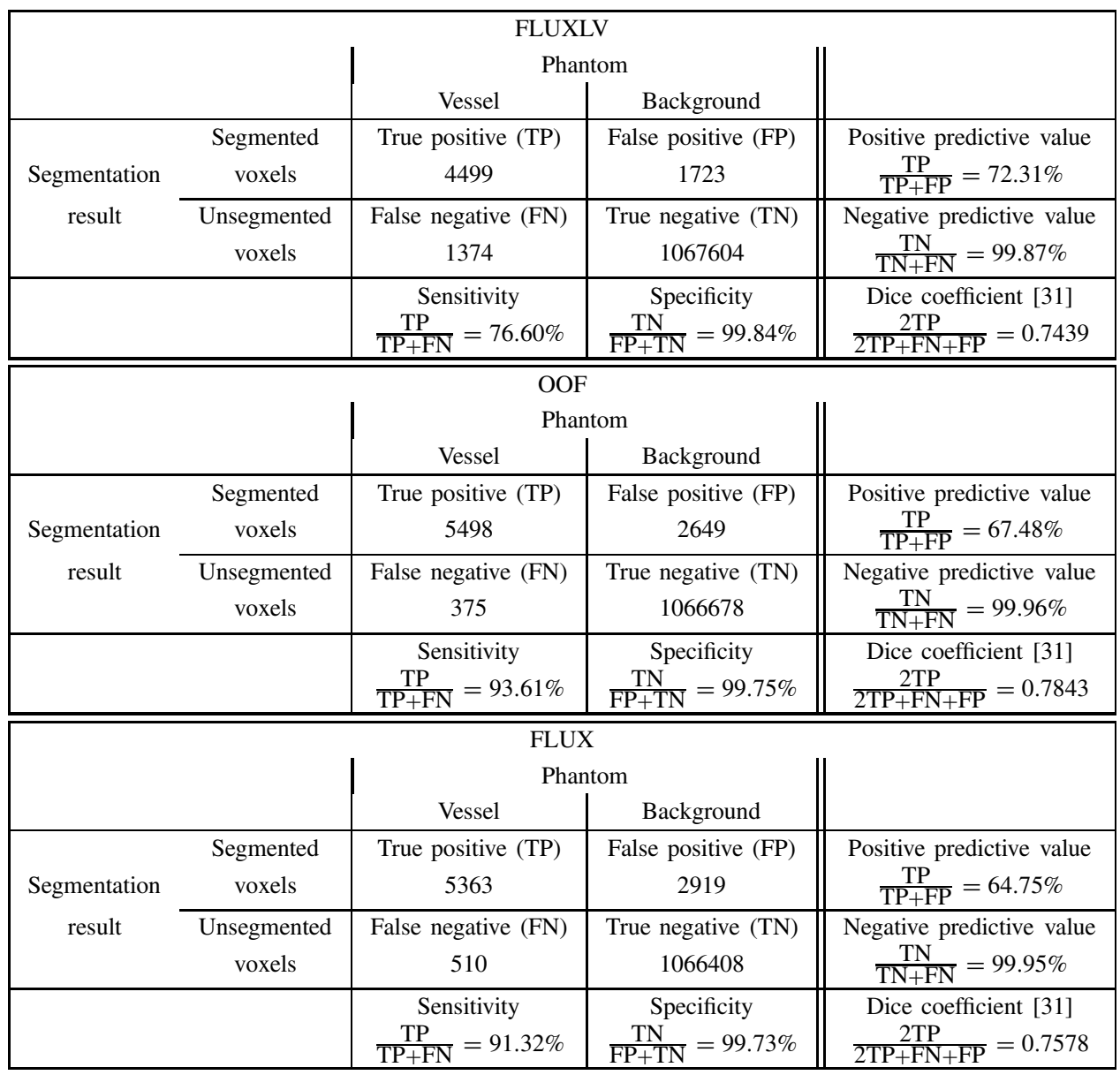

The former distance reveals how far the resultant contour overshoots the vessel boundary. The latter one quantifies the degree of under-segmentation. Either one of these distances is computed for each false positive voxel and each false negative voxel. The corresponding histograms are shown in Fig. 6. Due to the presence of the partial volume effect, the false positive or false negative cases with distance less than 1 voxel-length can be considered as satisfactory. From Fig. 6, over half of the false negative and the false positive cases occur within 1 voxel-length. The proposed method is therefore able to effectively segment the vessel and also the attached aneurysm. Analogous observation can be found on the distance histograms of FLUXLV, OOF and FLUX in Fig. 7(a)-(c). However, the histograms of FLUXLV, OOF and FLUX show more false negative voxels with distances larger than 2 voxel length. These false negative voxels correspond to the unsegmented aneurysmal regions.

The segmentation results of the four clinical PC-MRA images are studied in Figs. 8-16, analogous to the segmentation result of the vascular phantom shown in Figs. 3-5. In these clinical cases, the aneurysms locate at various positions and have different sizes and shapes. In Figs. 8(d), $10(\mathrm{~d}), 13(\mathrm{~d})$, and 15(d), the dimmest parts of the aneurysm domes are identified as the markers (middle columns) and subsequently recovered by the topology refinement process (right columns). The proposed method is capable of recovering the entire vascular structures, despite the contrast variation of vessels and the averse effect introduced by the turbulent flow inside aneurysms. Comparing the sagittal, axial and coronal views of the source images (Figs. 8(a), 10(a), 13(a) and 15(a)) and those of the segmentation result (Figs. 8(b), 10(b), 13(b) and 15(b)), the proposed method delivers promising segmentation results. On the contrary, the topology refinement process cannot locate any marker, hole or concave-cavity on the level set evolution results of FLUXLV, OOF and FLUX in the first, third and fourth cases. Comparing the first columns against the first to the third columns in Figs. 9(d), 14(d), and 16(d), FLUXLV, OOF and FLUX can capture noticeably smaller aneurysmal regions than the proposed method does. The evolving contours are halted undesirably inside the vasculatures. This creates large openings around the location where turbulent flows take place. It consequently prohibits any marker or hole to be discovered to reclaim the concavecavity in the topology refinement process. Nonetheless, if FLUXLV, OOF and FLUX segment enough voxels around the turbulent flow affected region, these three methods are capable of segmenting the aneurysms with the help of the topology refinement step. It is exemplified in their segmentation results in the aneurysmal regions of the second clinical case (see Fig. 12). 

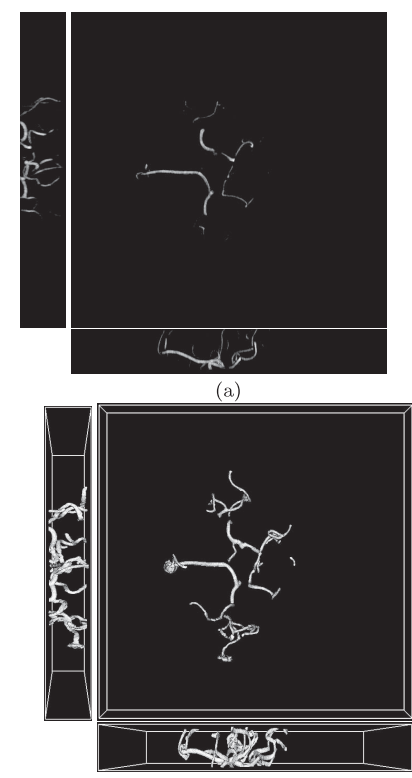

(b)
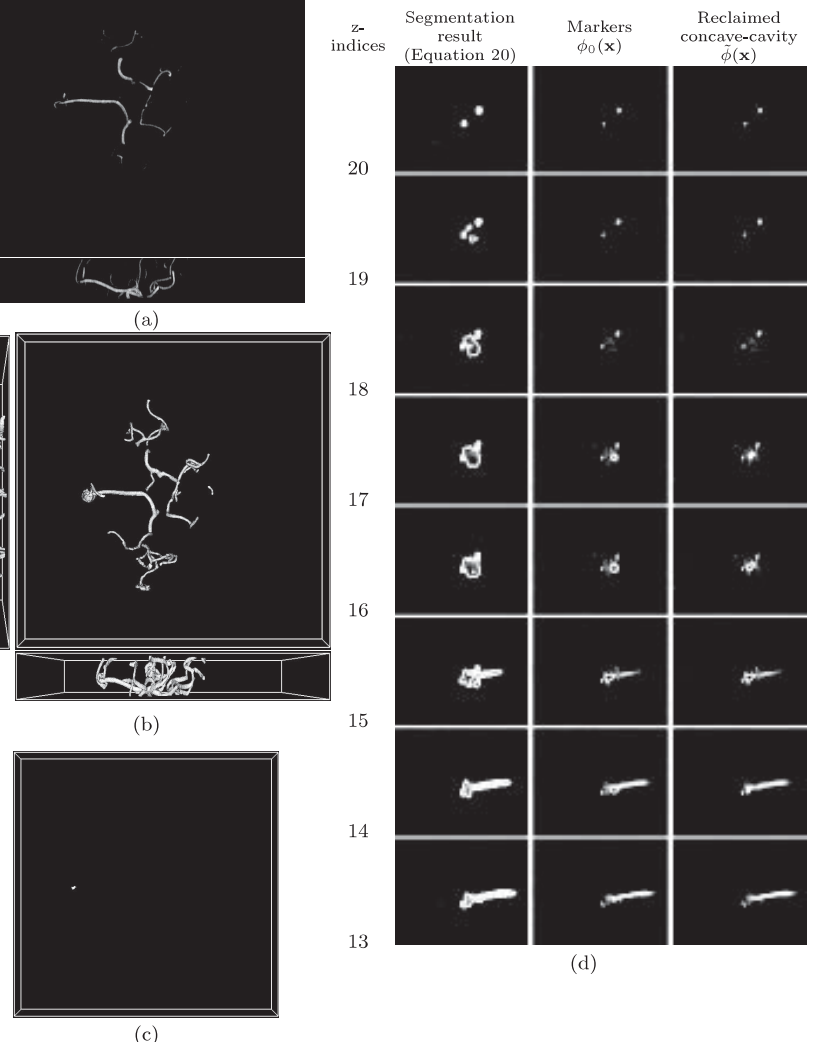

17

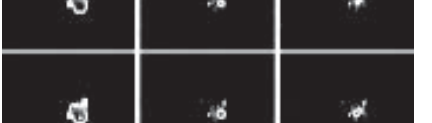

\section{.}

का

\section{${ }^{15}$}

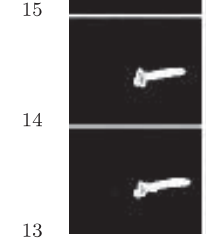

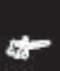

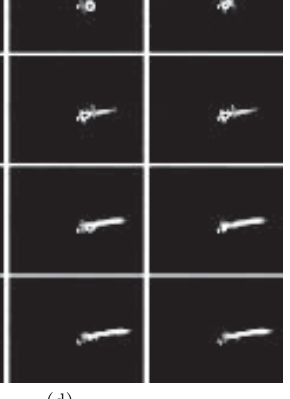

(d)

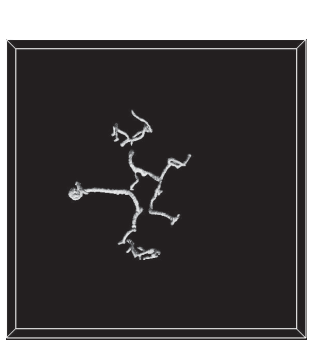

(a)

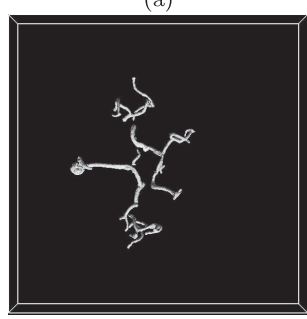

(b)

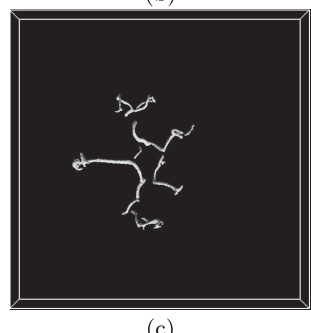

(c)

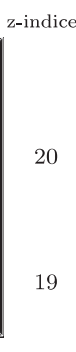

20

19

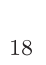

18

17

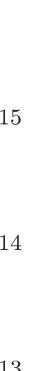

13

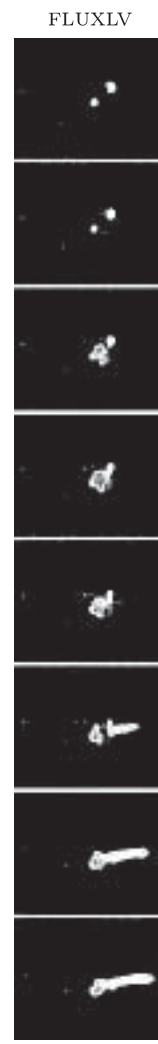

$\mathrm{OOF}$
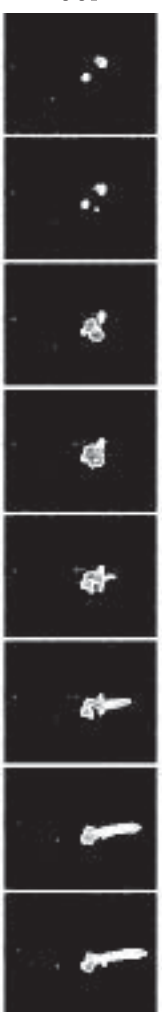

(d)
FLUX

Fig. 14. Experiment on the third clinical case. The final segmentation results (20) of different methods based on the image volume [Fig 13(a)] are depicted: (a) FLUXLV; (b) OOF; and (c) FLUX. (d) Zoomed-in images of the aneurysmal region of various methods, topology refinement cannot segment additional region for FLUXLV, OOF and FLUX in this case.

At an object boundary, the detection responses of the discontinuity descriptors based on a small detection region are weak because the intensity change occurs merely at the center of the region. The multiscale detection employed by OOF and FLUX as suggested in [16] and [30] in turn favors large detection region in order to search for strong intensity changes. The enlarged detection region possibly captures the intensity changes occurred across the vessel boundary (especially for a high contrast vessel boundary) in the vicinity of the local voxel, instead of the intensity changes at the local voxel. Without the use of local intensity variance to penalize an oversized detection sphere, the discontinuity descriptors of OOF and FLUX are more likely to report the voxels around the vessel boundaries as inside the vessels. This causes OOF and FLUX slightly overshoot the object boundary in 1 or 2 voxel-length.

2) Distilled Intensity Discontinuity Descriptor and Spherical Intensity Discontinuity Descriptor: Recognizing intensity changes across a spherical region boundary is one of the major components of the four examined approaches. In the proposed method, the distilled intensity discontinuity descriptor is chosen over its spherical counterpart for detection of non-spherical objects, such as aneurysms and vessels. The examined methods can be categorized as "distilled intensity discontinuity descriptor based methods" (the proposed method and $\mathrm{OOF}$ ) and "spherical intensity discontinuity descriptor based methods" (FLUXLV and FLUX). The latter methods are 

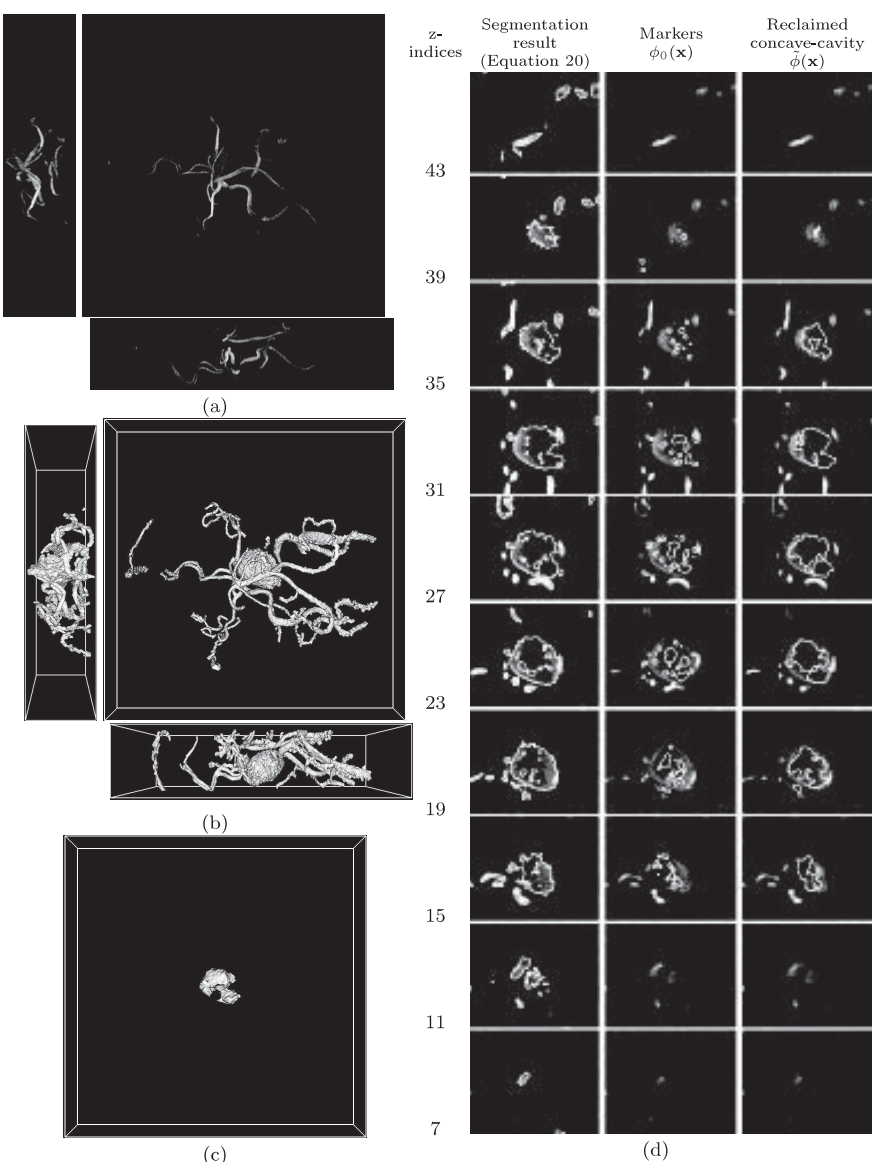

Fig. 15. Experiment on the fourth clinical case, an aneurysm at the internal carotid artery (ICA). (a) PC-MRA image volume (voxel size: $0.4 \times 0.4 \times 0.8 \mathrm{~mm}^{3}$, image dimension: $512 \times 512 \times 62$ voxels) used in the clinical data experiment. First row: sagittal and axial projections; second row: coronal projection. (b) Segmentation result. (c) Reclaimed holes and concave-cavities, which also suggest the aneurysm position. (d) Zoomed-in images of the aneurysmal region. The segmentation result is obtained by using $R=\{0.4,0.8,1.2,1.6,2.0,2.4,2.8,3.2,3.6,4.0,4.4,4.8\} \mathrm{mm}, D=$ $\{0.2,0.4,0.6,0.8,1.2,1.4,1.6,1.8,2.0,2.2,2.4,2.6,2.8,3.0,3.2\} \mathrm{mm}$. The initial seed points of the level set function $\psi$ are obtained by thresholding the brightest $0.1 \%$ voxels.

specifically designed to penalize deviations from tubularity and eccentric positions, and are thus well adapted to frameworks such as centerline detection and tracking. However, its major shortcoming is its consideration of intensity changes across the entire detection region boundary. It reduces detection response magnitude when vessel intensity drops along the structure. This can discourage contours propagating into intensity fluctuating vasculatures, such as aneurysms.

This observation is reflected by the fact that the distilled intensity discontinuity descriptor based methods can segment more vessels than their counterparts in some cases, for example.

1) The true positive values of the proposed method (Table II) is higher than that of FLUXLV (Table III), and the true positive values of OOF is higher than that of FLUX (Table III);

2) OOF segments significantly more vessels than FLUX does in the first, the second and the third clinical cases (compare Fig. 9(b) against Fig. 9(a), Fig. 11(b) against Fig. 11(a) and Fig. 14(b) against Fig. 14(a));

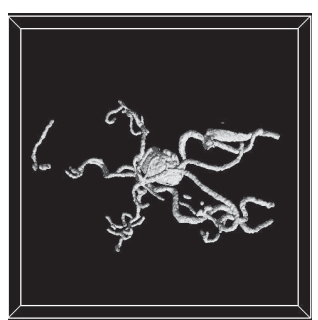

(a)

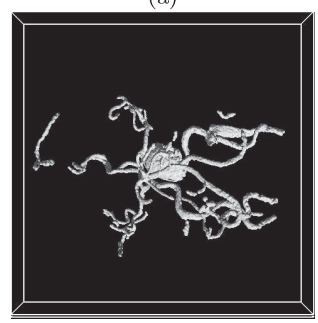

(b)

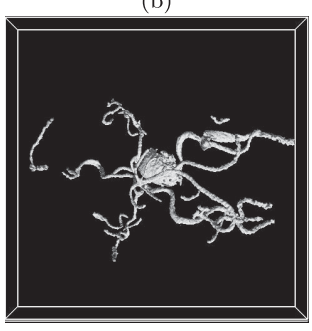

(c)

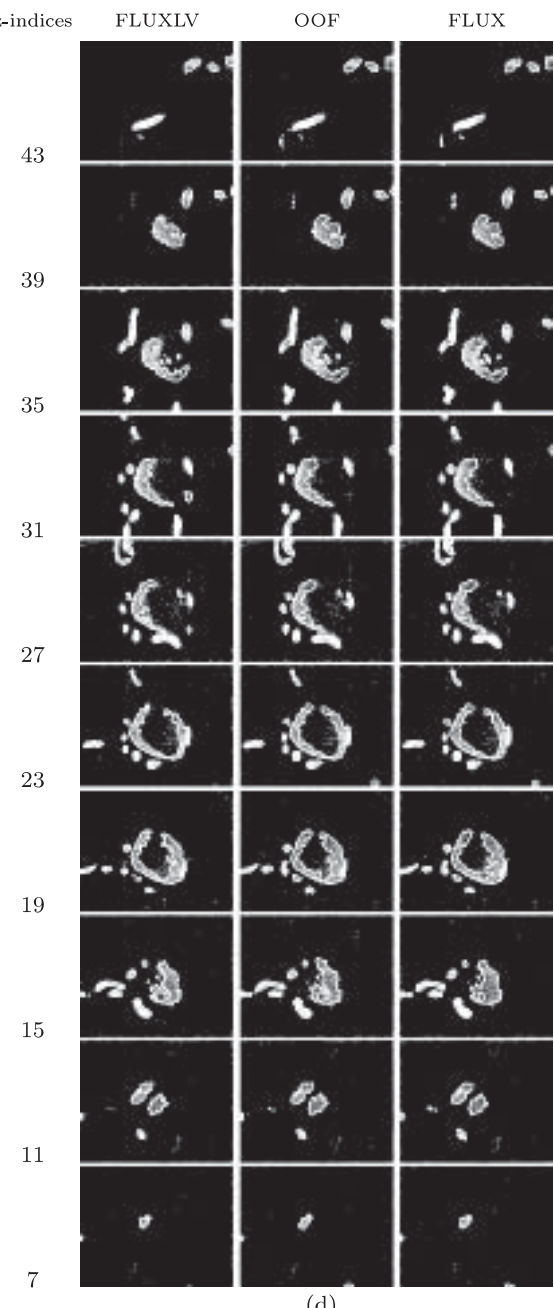

7

(d)

Fig. 16. Experiment on the fourth clinical case. The final segmentation results (20) of different methods based on the image volume [Fig. 15(a)] are depicted: (a) FLUXLV; (b) OOF; and (c) FLUX. (d) Zoomed-in images of the aneurysmal region of various methods, topology refinement cannot segment additional region for FLUXLV, OOF, and FLUX in this case.

3) the proposed method captures noticeably more vessels than FLUXLV does in the third and the fourth clinical cases (compare Figs. 13(b) against 14(c) and Figs. 15(b) against 16(c)).

3) DH Ratio Without Offset: Severe contour leakages are found when the DH ratio without offset (Equation 2) is employed in the segmentation framework. As discussed in Section II-A, it is over-sensitive to small intensity fluctuation. The leaked contours are not assessable and they are therefore not presented in this paper.

4) Summary: The distilled intensity discontinuity descriptor is less restrictive in the detection of spherical structures. It is therefore more flexible to handle shape variation of aneurysms. On the other hand, the offset DH ratio helps avoid contour leakages. It also suppresses the responses induced by the intensity changes inside aneurysms to facilitate the discovery of the aneurysmal regions. Employing both of them yields the best image features for intensity based segmentation of vessels and aneurysms in PC-MRA images. Therefore, the proposed method exhibits all advantages shown in Sections IV-D.1 and 
IV-D.2. It is noted that the subsequent refinement for turbulent flow affected regions consider no intensity information. An accurate refinement solely relies on the fact that the vicinity of those regions has been well discovered by the preceding segmentation procedure. A satisfactory intensity based segmentation algorithm is extremely crucial for the success of the topology refinement.

\section{CONCLUSION}

We have presented a novel approach to segmenting intracranial vessels and intracranial aneurysms on PC-MRA image volumes. The proposed method comprises two intensity based image features - the intensity discontinuity descriptor and local intensity variance to handle the low contrast structures. They jointly deliver the intensity discontinuity-homogeneity (DH) ratio for the identification of vasculatures. The final $\mathrm{DH}$ ratio (the offset and distilled DH ratio) is robust against vascular structures of varying sizes and shapes, and voxel intensity fluctuation. A subsequent topology refinement mechanism is devised to recover the turbulent flow-affected aneurysmal regions that are undetected by the $\mathrm{DH}$ ratio based segmentation scheme.

Considering the limited spatial resolution, low signal-tonoise ratio, flow orientation-dependent sensitivity (less sensitive to flows along the in-plane direction), the presence of ghost artifacts and turbulent flow of the current PCMRA technique, retrieving quantitative measures of aneurysms from PC-MRA images are challenging. With the aid of the proposed segmentation method, clinicians will be able to perform preliminary and non-invasive diagnosis using PCMRA images. Nonetheless, it is necessary to obtain more datasets along with corresponding manual segmentation to clinically validate the proposed method. Therefore, additional clinical data acquisition for extensive evaluation is the utmost important future direction of this research. Furthermore, in practice, small distal vessels might be out of interest for arterial aneurysm detection. When the small vessels which are disconnected from the major arteries can be omitted, an automatic initialization scheme is realized by thresholding a very small portion (for instance, $0.01 \%$ ) of highest intensity voxels from the PC-MRA image. This automatic scheme will ensure that there are seed points only inside the vessel component connected to the major high intensity arteries. Studying the clinical relevance of the segmentation results produced from this automatic initialization scheme will be another interesting direction of this work.

In this study, the proposed method is evaluated by utilizing an image volume with vascular phantom, and four clinical cases. Based on the same segmentation framework, various components of the proposed method - spherical intensity discontinuity descriptor, distilled intensity discontinuity descriptor, the associated $\mathrm{DH}$ ratios and topology refinement are examined. The proposed method is also compared against three existing vascular segmentation techniques. We inspect the weaknesses of the existing techniques and reason every component involved in the development of the proposed method. It is demonstrated that the proposed model is capable of selecting low intensity aneurysms of various sizes and shapes along with the blood vessels. It has experimentally shown that the proposed method can deliver promising results in the segmentation of aneurysms in PC-MRA images.

\section{APPENDIX}

\section{A. Expected Value of the Local Intensity Variance}

Given the voxel intensity is an i.i.d. variable, and $x_{i}$ is the $i$ th voxel sample in a local region consist of $n$ voxels. Without loss of generality, each voxel sample has a weight $\alpha_{n}(i)$ for the computation of the local intensity variance where $\sum_{i}^{n} \alpha_{n}(i)=$ 1. The expected value of the local intensity variance is,

$$
\begin{aligned}
& E\left(\sum_{i}^{n} \alpha_{n}(i) x_{i}^{2}-\left(\sum_{i}^{n} \alpha_{n}(i) x_{i}\right)^{2}\right) \\
& =E\left(x^{2}\right)\left(1-\sum_{i}^{n} \sum_{j}^{n} \alpha_{n}(i) \alpha_{n}(j)\right) .
\end{aligned}
$$

If all $n$ voxel samples receive the same weight, i.e. $\alpha_{n}(i)=$ $n^{-1}, \forall i \in[1, n]$, the expected local intensity variance is strictly monotonic increasing with respect to the sample size $n$.

\section{B. Computation of the Distilled Discontinuity Descriptor}

By the divergence theorem, the response of the distilled intensity discontinuity descriptor with a smoothed step function is,

$$
\frac{1}{4 \pi r^{2}} \int_{\Omega} d_{r}(\vec{v})\left[\vec{\nabla} \cdot\left(\hat{q}_{\vec{x}, r}^{1} \frac{\partial}{\partial \hat{q}_{\vec{x}, r}^{1}} I(\vec{x}+\vec{v})\right)\right] d \vec{v} .
$$

This equation can also be evaluated in the Fourier domain. The optimal direction $\hat{q}_{\vec{x}, r}^{1}$ is also computed analytically. It is accomplished by realizing the relation between $h_{r}(\vec{x})$ to the work of Optimally Oriented Flux [30]. Their relation is revealed by rewriting the right hand side of Equation 22 as,

$$
\frac{1}{4 \pi r^{2}} \int_{\partial S(\vec{x}, r)}\left(\left(\vec{\nabla}(g * I)\left(\vec{x}-r \hat{n}_{A}\right) \cdot \hat{q}_{\vec{x} ; r}^{1}\right) \hat{q}_{\vec{x} ; r}^{1}\right) \cdot \hat{n}_{A} d A .
$$

The maximal and minimal operations taken in Equation 8 are equivalent to the evaluation of optimally oriented flux and are calculated analytically by performing eigen-decomposition in a 3-by-3 symmetric tensor for each voxel in each detection range. Denote $i$ th basis vector in the three dimensional space by $\hat{y}_{i}$ and $i, j \in\{1,2,3\}$, the element of the symmetric tensor at the $i$ th row- $j$ th column is,

$$
\frac{1}{4 \pi r^{2}} \int_{\partial S_{r}}\left(\vec{\nabla}(g * I)\left(\vec{x}-r \hat{n}_{A}\right) \cdot \hat{y}_{i}\right)\left(\hat{n}_{A} \cdot \hat{y}_{j}\right) d A .
$$

The above equation represents six independent components for a symmetric 3-by-3 tensor. The construction of this tensor is accomplished as convolution operations in the spatial domain and computed in the Fourier domain [30]. It is analogous to the computation of Equation 12. Our FFT implementation employs the FFTW library [32]. Input images are mirror-padded by $\lceil 2 r /$ Voxel-size $\rceil$ voxels on each border (i.e. $2 \times\lceil 2 r /$ Voxel-size $\rceil$ voxels along each dimension). 


\section{REFERENCES}

[1] R. McLaughlin and J. Noble, "Demarcation of aneurysms using the seed and cull algorithm," in Proc. Int. Conf. Med. Image Comput. Comput. Assisted Intervent., 2002, pp. 419-426.

[2] Y. Sato, S. Nakajima, N. Shiraga, H. Atsumi1, S. Yoshida, T. Koller, G. Gerig, and R. Kikinis, "Three-dimensional multi-scale line filter for segmentation and visualization of curvilinear structures in medical images," Med. Image Anal., vol. 2, no. 2, pp. 143-168, 1998.

[3] M. Hernandez and A. Frangi, "Non-parametric geodesic active regions: Method and evaluation for cerebral aneurysms segmentation in 3DRA and CTA," Med. Image Anal., vol. 11, no. 3, pp. 224-241, 2007.

[4] H. Bogunović, J. Pozo, and M. Villa-Uriol, "Automated segmentation of cerebral vasculature with aneurysms in 3DRA and TOF-MRA using geodesic active regions: An evaluation study," Med. Phys., vol. 39, no. 1, pp. 210-222, 2011.

[5] V. Caselles, R. Kimmel, and G. Sapiro, "Geodesic active contours," Int. J. Comput. Vis., vol. 22, no. 1, pp. 61-79, 1997.

[6] A. Firouzian, R. Manniesing, H. Flach, R. Risselada, F. van Kooten, M. Sturkenboom, A. van der Lugt, and W. Niessen, "Intracranial aneurysm segmentation in 3D CT angiography: Method and quantitative validation with and without prior noise filtering," Eur. J. Radiol., vol. 79, no. 2, pp. 299-304, 2011.

[7] W. Wong and A. Chung, "Augmented vessels for quantitative analysis of vascular abnormalities and endovascular treatment planning," IEEE Trans. Med. Imag., vol. 25, no. 6, pp. 665-684, Jun. 2006.

[8] S. Yu, W. So, A. Chung, K. Lee, and G. Wong, "A compartmentalized volumetric system for outcome analysis of coiled cerebral aneurysms: Aneurysm-coil mass-neck outcome assessment system," Neurosurgery, vol. 64, no. 1, pp. 149-154, 2009.

[9] D. L. Wilson and J. A. Noble, "An adaptive segmentation algorithm for time-of-flight MRA data," IEEE Trans. Med. Imag., vol. 18, no. 10, pp. 938-945, Oct. 1999.

[10] A. Chung, J. Noble, and P. Summers, "Fusing speed and phase information for vascular segmentation of phase contrast MR angiograms," Med. Image Anal., vol. 6, no. 2, pp. 109-128, 2002.

[11] D. Lesage, E. Angelini, I. Bloch, and G. Funka-Lea, "A review of $3 \mathrm{D}$ vessel lumen segmentation techniques: Models, features and extraction schemes," Med. Image Anal., vol. 13, no. 6, pp. 819-845, 2009.

[12] M. de Bruijne, B. van Ginneken, M. Viergever, and W. Niessen, "Interactive segmentation of abdominal aortic aneurysms in CTA images," Med. Image Anal., vol. 8, no. 2, pp. 127-138, 2004.

[13] M. de Bruijne, B. van Ginneken, L. Bartels, M. van der Laan, J. Blankensteijn, W. Niessen, and M. Viergever, "Automated segmentation of abdominal aortic aneurysms in multi-spectral MR images," in Proc. Int. Conf. Med. Image Comput. Comput. Assisted Intervent., Feb. 2003, pp. 538-545.

[14] Q. Lin, "Enhancement, extraction, and visualization of 3D volume data," Ph.D. thesis, Dept. Electr. Eng., Linkoping Univ., Linkoping, Sweden, 2003.

[15] M. Law and A. Chung, "Vessel and intracranial aneurysm segmentation using multi-range filters and local variances," in Proc. Int. Conf. Med. Image Comput. Comput. Assisted Intervent., 2007, pp. 866-874.

[16] A. Vasilevskiy and K. Siddiqi, "Flux maximizing geometric flows," IEEE Trans. Pattern Anal. Mach. Intell., vol. 24, no. 12, pp. 1565-1578, Dec. 2002.

[17] M. Law and A. Chung, "Efficient implementation for spherical flux computation and its application to vascular segmentation," IEEE Trans. Image Process., vol. 18, no. 3, pp. 596-612, Mar. 2009.

[18] K. Krissian, "Flux-based anisotropic diffusion applied to enhancement of 3-D angiogram," IEEE Trans. Med. Imag., vol. 21, no. 11, pp. 1440-1442, Nov. 2002.

[19] S. Bouix, K. Siddiqi, and A. Tannenbaum, "Flux driven automatic centerline extraction," Med. Image Anal., vol. 9, no. 3, pp. 209-221, 2005.

[20] M. Descoteaux, M. Audette, K. Chinzei, and K. Siddiqi, "Bone enhancement filtering: Application to sinus bone segmentation and simulation of pituitary surgery," Comput. Aided Surgery, vol. 11, no. 5, pp. 247-255, 2006.

[21] M. Descoteaux, D. L. Collins, and K. Siddiqi, "Multi-scale geometric flow for segmenting vasculature in proton-density weighted MRI," Med. Image Anal., vol. 12, no. 4, pp. 497-513, 2008.

[22] A. Frangi, W. Niessen, K. Vincken, and M. Viergever, "Multiscale vessel enhancement filtering," in Proc. Int. Conf. Med. Image Comput. Comput. Assisted Intervent., 1998, pp. 130-137.
[23] M. Law and A. Chung, "Weighted local variance based edge detection and its application to vascular segmentation in magnetic resonance angiography," IEEE Trans. Med. Imag., vol. 26, no. 9, pp. 1224-1241, Sep. 2007.

[24] R. Bracewell, The Fourier Transform and Its Application. New York: McGraw-Hill, 1986.

[25] R. Malladi, J. Sethian, and B. Vemuri, "Shape modeling with front propagation: A level set approach," IEEE Trans. Pattern Anal. Mach. Intell., vol. 17, no. 2, pp. 158-175, Feb. 1995.

[26] R. Whitaker, "A level-set approach to $3 \mathrm{D}$ reconstruction from range data," Int. J. Comput. Vis., vol. 29, no. 33, pp. 203-231, Sep. 1998.

[27] L. Ibanez, W. Schroeder, L. Ng, and J. Cates. The ITK Software ToolKit. (2005) [Online]. Available: http://www.itk.org

[28] H. Steiger, J. Oshinski, R. Pettigrew, and D. Ku, "Computational simulation of turbulent signal loss in 2D time-of-flight magnetic resonance angiograms," Magn. Reson. Med., vol. 37, no. 4, pp. 609-614, Apr. 1997.

[29] Y. Gobin, J. Counord, P. Flaud, and J. Duffaux, "In vitro study of haemodynamics in a giant saccular aneurysm model: Influence of flow dynamics in the parent vessel and effects of coil embolisation," Neuroradiology, vol. 36, no. 7, pp. 530-536, 1994.

[30] M. Law and A. Chung, "Three dimensional curvilinear structure detection using optimally oriented flux," in Proc. Eur. Conf. Comput. Vis., 2008, pp. 368-382.

[31] A. Zijdenbos, B. Dawant, R. Margolin, and A. Palmer, "Morphometric analysis of white matter lesions in MR images: Method and validation," IEEE Trans. Med Imag., vol. 13, no. 4, pp. 716-724, Dec. 1994.

[32] M. Frigo and S. Johnson, "The design and implementation of FFTW3," Proc. IEEE, vol. 93, no. 2, pp. 216-231, Feb. 2005.

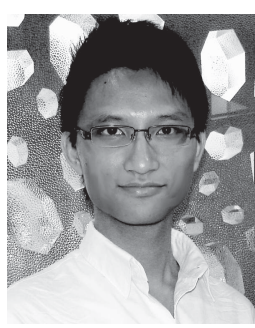

Max W. K. Law received the B.Eng. degree in computer engineering, the M.Phil. degree in computer science, and the Ph.D. degree in computer science and engineering from the Hong Kong University of Science and Technology, Kowloon, Hong Kong, in 2004, 2006, and 2010, respectively.

$\mathrm{He}$ was an Intern with Siemens Corporate Research, Princeton, NJ, in 2009. In collaboration with GE Healthcare, he is currently a Post-Doctoral Fellow with the Digital Imaging Group of London and the Department of Medical Biophysics, Western

University, London, ON, Canada. His current research interests include lowlevel vision, image processing, and medical image analysis.

Dr. Law was a recipient of the Mitacs-Accelerate Fellowship and the MitacsElevate Fellowship.

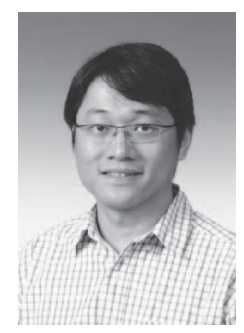

Albert C. S. Chung received the B.Eng. degree in computer engineering from the University of Hong Kong, Hong Kong, the M.Phil. degree in computer science from the Hong Kong University of Science and Technology, Kowloon, Hong Kong, and the Doctoral degree in computer or natural vision from the Medical Vision Laboratory, University of Oxford, Oxford, U.K., in 1995, 1998, and 2001, respectively.

He was a Visiting Scientist with the Artificial Intelligence Laboratory, Massachusetts Institute of Technology, Cambridge, in 2001. He is currently an Associate Professor with the Department of Computer Science and Engineering, Hong Kong University of Science and Technology. His current research interests include medical image analysis, image processing, and computer vision.

Dr. Chung was a recipient of the Croucher Foundation Scholarship and the British Machine Vision Association Sullivan Thesis Award in 2002 for the best doctoral thesis submitted to a U.K. university on computer or natural vision. 